\title{
Production and electromagnetic decay of hyperons: a feasibility study with HADES as a phase-0 experiment at FAIR
}

\author{
(HADES collaboration and PANDA@HADES collaboration)
}

J. Adamczewski-Musch ${ }^{4}$, A. Belyaev ${ }^{7}, \quad$ A. Blanco ${ }^{1}, \quad$ C. Blume ${ }^{8}$, C. Charlotte ${ }^{8}$, D. S. Borisenko ${ }^{12}$, C. Charlotte ${ }^{8}$, L. Chlad ${ }^{16}$, P. Chudoba ${ }^{16}$, I. Ciepał ${ }^{2}$, A. Derichs ${ }^{11}$, D. Dittert ${ }^{5}$, J. Dreyer ${ }^{6}$, W. Esmail ${ }^{11}$, O. Fateev ${ }^{7}$, P. Fonte ${ }^{1, a}$, J. Friese $^{9}$, I. Fröhlich ${ }^{8}$, J. Förtsch ${ }^{20}$, T. Galatyuk ${ }^{5,4}$, I. Georgadze ${ }^{11}$, O. Golosov ${ }^{14}$, M. Golubeva ${ }^{12}$, R. Greifenhagen ${ }^{6, b}$, M. Grunwald ${ }^{20}$, D. Grzonka ${ }^{11}$, F. Guber ${ }^{12}$, M. Gumberidze ${ }^{4}$, S. Harabasz ${ }^{5}$, T. Heinz ${ }^{4}$, B. Heybeck ${ }^{8}$, C. Höhne ${ }^{10,4}$, M. Holona ${ }^{11}$, R. Holzmann ${ }^{4}$, H. Huck ${ }^{8}$, A. Ierusalimov ${ }^{7}$, M. Imre ${ }^{15}$, A. Ivashkin ${ }^{12}$, B. Kämpfer ${ }^{6, b}$, K-H. Kampert ${ }^{20}$, B. Kardan ${ }^{8}$, V. Kedych ${ }^{5}$, V. Khomyakov ${ }^{13}$, I. Koenig ${ }^{4}$, W. Koenig ${ }^{4}$, M. Kohls ${ }^{8}$, G. Kornakov ${ }^{5}$, F. Kornas ${ }^{5}$, R. Kotte ${ }^{6}$, A. Kozela ${ }^{2}$, V. Kozlov ${ }^{11}$, I. Kres ${ }^{20}$, J. Kuboś ${ }^{2}$, A. Kugler ${ }^{16}$, P. Kulessa ${ }^{11}$, V. Ladygin ${ }^{7}$, R. Lalik ${ }^{3}$, C. Le Galliard ${ }^{15}$, A. Lebedev ${ }^{13}$, S. Lebedev ${ }^{10,7}$, S. Linev ${ }^{4}$, L. Lopes ${ }^{1}$, M. Lorenz ${ }^{8}$, G. Lykasov ${ }^{7}$, A. Malige ${ }^{3}$, J. Markert ${ }^{4}$, T. Matulewicz ${ }^{18}$, J. Michel ${ }^{8}$, S. Morozov ${ }^{12,14}$, C. Müntz ${ }^{8}$, L. Naumann ${ }^{6}$, K. Nowakowski ${ }^{3}$, S. Orfanitsky ${ }^{11}$, J. -H. Otto ${ }^{10}$, V. Patel ${ }^{20}$, C. Pauly ${ }^{20}$, V. Pechenov ${ }^{4}$, O. Pechenova ${ }^{4}$, G. Perez Andrade ${ }^{11}$, O. Petukhov ${ }^{12}$, D. Pfeifer ${ }^{20}$, K. Piasecki ${ }^{18}$, J. Pietraszko ${ }^{4}$, A. Prozorov ${ }^{16}$, W. Przygoda ${ }^{3}$, K. Pysz ${ }^{2}$, B. Ramstein ${ }^{15}$, N. Rathod ${ }^{3}$, J. Regina ${ }^{17}$, A. Reshetin ${ }^{12}$, S. Reznikov ${ }^{7}$, J. T. Rieger ${ }^{17}$, J. Ritman ${ }^{4,11}$, P. Rodriguez-Ramos ${ }^{16}$, A. Rost ${ }^{5}$, A. Rustamov ${ }^{4}$, P. Salabura ${ }^{3}$, J. Saraiva ${ }^{1}$, N. Schild ${ }^{5}$, E. Schwab ${ }^{4}$, K. Schönning ${ }^{17}$, F. Scozzi ${ }^{5,15}$, F. Seck ${ }^{5}$, I. Selyuzhenkov ${ }^{4,14}$, V. Serdyuk ${ }^{11}$, A. Shabanov ${ }^{12}$, U. Singh ${ }^{3}$, J. Smyrski ${ }^{3}$, M. Sobiella ${ }^{6}$, S. Spies ${ }^{8}$, M. Strikhanov ${ }^{14}$, H. Ströbele ${ }^{8}$, J. Stroth ${ }^{8,4}$, K. Sumara ${ }^{3}$, O. Svoboda ${ }^{16}$, M. Szala ${ }^{8}$, J. Szewczyk ${ }^{19}$, A. Taranenko ${ }^{14}$, P. Tlusty ${ }^{16}$, M. Traxler ${ }^{4}$, V. Wagner ${ }^{16}$, M. Wasiluk ${ }^{19}$, A. A. Weber ${ }^{10}$, C. Wendisch ${ }^{4}$, P. Wintz $^{11}$, B. Włoch $^{2}$, H. P. Zbroszczyk ${ }^{19}$, E. Zherebzova ${ }^{12}$, A. Zhilin ${ }^{13}$, A. Zinchenko ${ }^{7}$, P. Zumbruch ${ }^{4}$

${ }^{1}$ LIP-Laboratório de Instrumentação e Física Experimental de Partículas, 3004-516 Coimbra, Portugal

${ }^{2}$ Institute of Nuclear Physics, Polish Academy of Sciences, Kraków, Poland

${ }^{3}$ Smoluchowski Institute of Physics, Jagiellonian University of Cracow, Kraków, Poland

${ }^{4}$ GSI Helmholtzzentrum für Schwerionenforschung GmbH, Darmstadt, Germany

5 Technische Universität Darmstadt, Darmstadt, Germany

${ }^{6}$ Institut für Strahlenphysik, Helmholtz-Zentrum Dresden-Rossendorf, Dresden, Germany

${ }^{7}$ Joint Institute of Nuclear Research, Dubna, Russia

${ }^{8}$ Institut für Kernphysik, Goethe-Universität, Frankfurt, Germany

${ }^{9}$ Physik Department E62, Technische Universität München, Garching, Germany

${ }^{10}$ II.Physikalisches Institut, Justus Liebig Universität Giessen, Giessen, Germany

${ }^{11}$ Forschungszentrum Jülich, Jülich, Germany

12 Institute for Nuclear Research, Russian Academy of Science, Moscow, Russia

${ }^{13}$ Institute of Theoretical and Experimental Physics, Moscow, Russia

${ }^{14}$ National Research Nuclear University MEPhI (Moscow Engineering Physics Institute), Moscow, Russia

${ }^{15}$ Laboratoire de Physique des 2 infinis Irne Joliot-Curie, Universit Paris-Saclay, CNRS-IN2P3., Orsay, France

16 Nuclear Physics Institute, The Czech Academy of Sciences, Rez, Czech Republic

(W.A. Esmail, P. Kulessa, R. Lalik, A. Malige, K. Nowakowski, G. Perez Andrade, W. Przygoda, N. Rathod, J. Ritman, P. Salabura, U. Singh, J. Smyrski, P. Wintz: member of the HADES and PANDA@HADES collaborations).

(A. Derichs, I. Georgadze, M. Holona, V. Kozlov, S. Orfanitsky, J. Regina, J.T. Rieger, K. Schönning, V. Serdyuk: member of the PANDA and PANDA@HADES collaborations). 
17 Institutionen för fysik och astronomi, Uppsala universitet, Uppsala, Sweden

18 Uniwersytet Warszawski - Instytut Fizyki Doświadczalnej, Warszawa, Poland

19 Warsaw University of Technology, Warsaw, Poland

20 Bergische Universität Wuppertal, Wuppertal, Germany

Received: 15 October 2020 / Accepted: 4 February 2021 / Published online: 20 April 2021

(C) The Author(s) 2021

Communicated by Frank Maas.

Abstract A feasibility study has been performed in order to investigate the performance of the HADES detector to measure the electromagnetic decays of the hyperon resonances $\Sigma(1385)^{0}, \Lambda(1405)$ and $\Lambda(1520)$ as well as the production of double strange baryon systems $\Xi^{-}$and $\Lambda \Lambda$ in $p+p$ reactions at a beam kinetic energy of $4.5 \mathrm{GeV}$. The existing HADES detector will be upgraded by a new Forward Detector, which extends the detector acceptance into a range of polar angles that plays a crucial role for these investigations. The analysis of each channel is preceded by a consideration of the production cross-sections. Afterwards the expected signal count rates using a target consisting of either liquid hydrogen or polyethylene are summarized.

\section{Contents}

1 Introduction ................ . 2

2 HADES and the Forward Detector . . . . . . . 3

2.1 Straw tracking stations ......... 4

2.2 The FRPC time-of-flight detector . . . . . . . 5

2.3 Digitization and track reconstruction . . . . . 6

3 Benchmark channels ... . . . . . . . 7

3.1 Cross-section estimates ... . . . . . 8

3.1.1 Production cross-sections of excited hyperons . 8

3.1.2 Decay branching ratios of excited hyperons 9

3.1.3 Production of double strangeness . . . . 9

3.1.4 Background channels . . . . . . . . . . . 9 9

4 Simulation and analysis techniques . . . . . . . 10

4.1 Acceptance for hyperon decays . . . . . . . . . 10

4.2 Trigger filtering . . . . . . . . . . . . 11

4.3 Track reconstruction and particle identification . 12

4.4 Inclusive $\Lambda$ reconstruction . . . . . . . . . 12

5 Results ................. . 14

5.1 Exclusive real photon decay of hyperons . . . . 14

5.2 Dalitz-decay of excited hyperons . . . . . . 16

5.3 Inclusive $\Xi^{-}$production . . . . . . . . . 17

$5.4 \Lambda \Lambda$ production ................. 19

5.5 Count rate estimates . . . . . . . . . . . . 19

6 Summary ................ 20

a. Also at Coimbra Polytechnic - ISEC, Coimbra, Portugal

b. Also at Technische Universität Dresden, Dresden, Germany

* e-mail: hades-info@gsi.de (Corresponding author)

\section{Introduction}

Using N-N and $\pi-\mathrm{N}$ reactions, the High Acceptance DiElectron Spectrometer (HADES [1]) explores the electromagnetic structure of baryonic resonances [2,3] and investigates their production mechanisms for beam kinetic energies in the region of a few GeV [4-7]. These studies also establish important reference data for the interpretation of heavy ion induced reactions, as demonstrated in the recent measurement of dilepton emission from dense baryonic matter [8]. A common aspect for these reactions is the need to understand the coupling of baryons with virtual (massive) photons. These couplings can be studied in proton and pion induced reactions by measuring the Dalitz-decay of baryonic resonances (e.g. $\mathrm{N}^{*}, \Delta$ ) into $\mathrm{Ne}^{+} \mathrm{e}^{-}$. These transitions provide valuable information on electromagnetic Transition Form Factors of baryonic resonances (eTFF) in the timelike kinematic region, e.g. $\Delta(1232) \rightarrow \mathrm{Ne}^{+} \mathrm{e}^{-}[2]$.

In general, eTFFs are analytical functions of the squared four-momentum transfer $\left(q^{2}\right)$ of the virtual photon exchanged between the initial and final state baryon. They are studied in the spacelike $\left(q^{2}<0\right)$ and the timelike $\left(q^{2}>0\right)$ kinematic regions in electron scattering off the nucleon and in Dalitz-decays, respectively. In the latter case, the virtual photon decays to a lepton pair (dielectron or dimuon) with an invariant mass $M$ within the region of $4\left(M_{l}\right)^{2}<M^{2}<$ $\left(M_{\mathrm{R}}-M_{\mathrm{N}}\right)^{2}$, where $M_{l}, M_{\mathrm{R}}$ and $M_{\mathrm{N}}$ are the masses of the lepton, the decaying resonance and the nucleon, respectively. Note that $q^{2}=0$ corresponds to the radiative decay i.e. emission of a real photon. Results published by HADES for nonstrange baryons indicate a significant role in the transitions for an intermediate $\rho$ vector meson, in agreement with the Vector Meson Dominance model [2,9]. Microscopic calculations based on the covariant spectator quark model explain this strong coupling to the meson as predominately an effect of the pion cloud in the nucleon $[10,11]$. This conclusion complements findings from electro-scattering experiments, where meson cloud effects are important for descriptions of the eTFF in the low $q^{2}$ spacelike region for several baryon resonances (for an overview see [12]).

Experimental results on hyperon radiative decays are still very sparse, despite their importance being stressed already many years ago. In the 1980's the radiative decay widths were discussed as an ideal observable to differentiate between various quark, bag and soliton models 
of strange baryons [13]. Since then, only a few measurements of the decays $\Lambda(1520) \rightarrow \Lambda \gamma, \Sigma(1385)^{0} \rightarrow \Lambda \gamma$ and $\Sigma(1385)^{+} \rightarrow \Sigma^{+} \gamma$ have become available [14-16]. The results on $\Sigma(1385)^{0} \rightarrow \Lambda \gamma$ decouplet-octet transitions are particularly interesting in view of the analogy within $\mathrm{SU}(3)$ flavor symmetry to $\Delta \rightarrow \mathrm{N} \gamma$, both of which are dominated by a magnetic dipole transition [17]. The measured decay widths are found to be almost a factor 2 larger than most quark model predictions. This finding is interpreted as evidence for effects of the pion cloud.

In the timelike region, the CLEO collaboration provided the first measurement of hyperon $\left(\Lambda, \Sigma^{ \pm, 0}, \Xi^{-, 0}\right.$ and $\Omega$ ) elastic magnetic form factors in $\mathrm{e}^{+} \mathrm{e}^{-}$annihilation data at large momentum transfers of $q^{2}=14.2 \mathrm{GeV} / c^{2}$ [18]. The form factors for the isoscalar $\Lambda$ state are a factor 1.7(2) larger than for the isovector $\Sigma^{0}$. This has been interpreted as evidence of correlation between light quarks in the hyperon. Indeed, one expects that light quarks couple to spin $S=0$ for the $\Lambda$ and $S=1$ for the $\Sigma^{0}$. Up to now, there are no measurements of eTFF in Dalitz-decays of hyperons. Since Dalitz-decays probe hyperon structure at lower $q^{2}$, mesonic degrees of freedom are expected to play an important role, just as for the non-strange baryons. Moreover, this $q^{2}$ domain allows for a more direct connection to the spacelike region where some data are available. Indeed, theoretical calculations based on dispersion relations [19] or quark models [20,21] provide predictions for decouplet-octet transitions in both the timelike and spacelike regions. In particular, recent calculations on $\Sigma(1385)^{0} \rightarrow \Lambda \mathrm{e}^{+} \mathrm{e}^{-}$show, in analogy to $\Delta \rightarrow \mathrm{Ne}^{+} \mathrm{e}^{-}$, significant effects of the pion cloud in the distribution of dilepton invariant mass [21]. As mentioned above, pion cloud effects have already been suggested to explain the large decay width for the $\Sigma^{* 0} \rightarrow \Lambda \gamma$ transition [16]. Also, calculations based on an effective Lagrangian of vector-baryon interactions predict significant effects of intermediate vector mesons $(\rho / \omega / \phi)$ for hyperon transitions [22,23]. Therefore, first measurements at HADES on both virtual (i.e. dielectron) and real photon decays: $\Sigma^{*} / \Lambda^{*} \rightarrow \Lambda \mathrm{e}^{+} \mathrm{e}^{-}(\gamma)$, respectively, will significantly impact our understanding of the electromagnetic structure of strange resonances in the $q^{2}$ region, where vector meson effects are expected to be large [23].

In addition, the collected data will enable detailed studies of hyperon production in proton-proton reactions and provide important reference measurements for future heavy-ion experiments exploring the high net-baryon density region of the QCD phase diagram (e.g. FAIR, NICA, STAR-BES). In particular, essentially nothing is known about the production of hyperons with masses larger than the $\Lambda$ and $\Sigma^{0}$ and of multi-strange hyperons in this beam energy range. The impact of the higher lying hyperon resonances on QCD thermodynamics, on freeze-out in heavy ion collisions and on the evolution of the early universe has been recently reported [24]. Measurements of the respective excitation functions in
$\mathrm{p}+\mathrm{A}$ and $\mathrm{A}+\mathrm{A}$ collisions are planned with the $\mathrm{CBM}$ detector [25]. HADES measurements will therefore provide a necessary reference to quantify the expected strangeness enhancement in heavy ion collisions and cold matter effects. One prominent example of the impact of such reference measurements is the puzzling enhancement of $\Xi$ cascade production in $\mathrm{Ar}+\mathrm{KCl}$ reactions at $1.75 \mathrm{~A} \mathrm{GeV}$ observed by the HADES collaboration. It was shown that the measured cascade yield is higher by a factor 10 to 100 than predicted in various theoretical models [26]. A similar enhancement observed in the $\mathrm{p}+\mathrm{Nb}$ system points to effects appearing already in cold nuclear matter [27]. Production on correlated nucleon pairs or excitation of higher mass resonances with a significant decay branch to $\Xi K K$ have been considered as possible explanations $[28,29]$. This reaction is just one example illustrating the importance of the data on double-strange hyperon production in nucleon-nucleon interactions close to the production threshold. Similarly, little or no data exist on the production of higher mass $(\Sigma(1385))$ or excited hyperon states (for example $\Lambda(1520)$ ) [30].

An important aspect of the double-strangeness production program is double $\Lambda$ production. The $\Lambda-\Lambda$ measurement is part of the more general baryon-baryon (p-p, p- $\Lambda, \Lambda$ $\Lambda$ ) correlation studies [31]. These data will constrain the rather poorly known hyperon-hyperon interaction, which has a key role in $\Lambda-\Lambda$ double hypernuclei, neutron star core studies [32] and the $\Xi^{-}$production mechanism. The proposed measurement will complement upcoming studies by the PANDA collaboration of $\Lambda-\Lambda$ and $\Lambda-\bar{\Lambda}$ in $p+p$ and $\mathrm{p}+\overline{\mathrm{p}}$ interactions, respectively [33].

Our paper is organized as follows. Section 2 includes a brief overview of HADES and the Forward Detector upgrade essential for the hyperon physics program outlined above, including detector simulations and test beam results. In Sect. 3, we present selected benchmark channels in order to demonstrate the feasibility of HADES to measure radiative decays of hyperons and to investigate double-strangeness production. This is followed in Sect. 4 by a description of the simulation and analysis steps, starting with $\Lambda$ reconstruction, which is a common step in all analyses presented here. Thereafter in Sect. 5 the channel-specific procedures are presented. Finally, estimated count rates are presented as well as a summary of this work and an outlook in Sect. 6.

\section{HADES and the Forward Detector}

All measurements discussed here can be performed with proton beams provided by the SIS18 with energies up to $E_{\text {kin }}=4.5 \mathrm{GeV}$ at HADES. HADESis a magnetic spectrometer that is in operation since 2002 at the SIS18 accelerator in the GSI Helmholtz Center for Heavy Ion Research in Darmstadt (Germany) [1]. It measures charged hadrons 


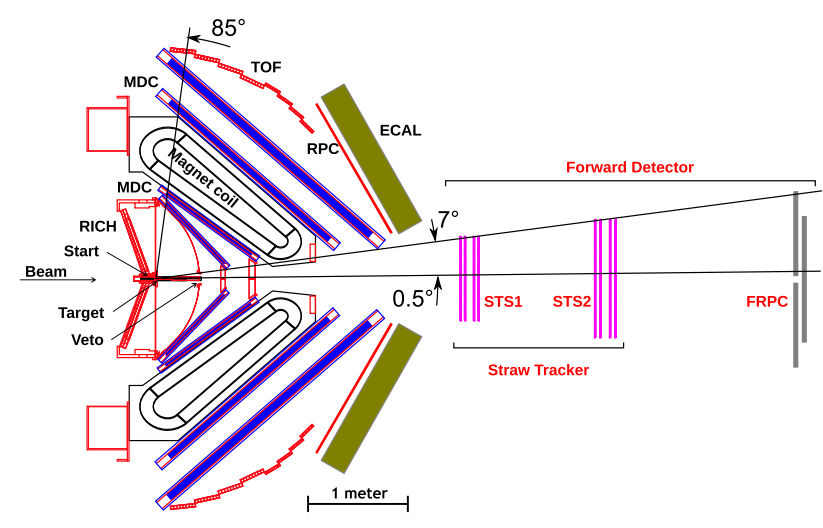

Fig. 1 Schematic cross-sectional view of the HADES spectrometer. The new Forward Detector extends the angular coverage into the lower polar angle range $0.5^{\circ}$ to $7^{\circ}$. The experiments described here also profit from the newly installed ECAL and upgraded RICH detectors

(pions, kaons and protons), leptons (electrons and positrons) and photons resulting from proton, secondary pion and heavy ion induced reactions on fixed proton or nuclear targets in the energy regime of a few AGeV. It features an excellent invariant mass resolution for electron-positron pairs of $\Delta M / M \approx 2.5 \%$ in the $\rho / \omega / \phi$ vector meson mass region. With its versatility it is an excellent tool to study the properties of hadrons in vacuum, cold and also dense baryonrich matter at moderate temperatures (for a recent review see [34]). HADES thus provides a lower energy reference for the future NICA and FAIR facilities and complements the region of high temperatures and small or even vanishing net baryon densities probed by experiments at SPS, RHIC and LHC. Results obtained in the last decade by HADES showed that baryonic resonances have a fundamental role in the processes that define the physics at finite baryon density. Since resonances are such important sources for meson production in heavy ion collisions at kinetic energies of a few $\mathrm{GeV}$, these systems are often described as "resonance matter" [35].

Future HADES operation within the new experimental facility of FAIR [36], will enable reactions to be measured with proton beam energies up to $29 \mathrm{GeV}$, a region where string fragmentation becomes dominant. To prepare for these new experimental challenges, the HADES spectrometer is currently undergoing several hardware upgrades: the Ring Imaging Cherenkov ( $\mathrm{RICH}$ ) photon detector has been upgraded to improve the dilepton identification, and an Electromagnetic Calorimeter (ECAL) has been installed for gamma reconstruction. Both components have successfully been used in 2019. Furthermore, new and faster readout electronics (DAQ) as well as a Forward Detector, to add particle tracking and velocity measurements in the forward direction, are currently being installed. These new upgrades are expected to increase the count rate by a factor of 50-100 for many of the interesting channels. In particular, the new setup will enable investigations of the production of hyperon states with single and double strangeness and their rare radiative decays and is a part of the FAIR Phase-0 program, in which the upgraded SIS18 accelerator is being used together with those components of the FAIR experiments that have already been completed. A schematic drawing of the upgraded setup is shown in Fig. 1.

The Forward Detector has full azimuthal coverage for polar angles in the range $0.5^{\circ}$ to $7^{\circ}$. It consists of two Straw Tracking Stations (STS1, STS2), which are based on instrumentation developed for PANDA [37,38], and a Forward Resistive Plate Chamber (FRPC) detector for Timeof-Flight (ToF) measurements. These detectors are located $3.1 \mathrm{~m}, 4.6 \mathrm{~m}$ and $7.5 \mathrm{~m}$ downstream of the target, respectively. The detectors are described in more detail below.

The maximum proton beam intensity is limited to $7.5 \times$ $10^{7} \mathrm{p} / \mathrm{s}$ by the HADES start detector. The existing HADES DAQ can readout a maximum trigger rate of $50 \mathrm{kHz}$, which is expected for this beam intensity incident on the HADES liquid hydrogen $\left(\mathrm{LH}_{2}\right)$ target and using the envisaged trigger setting (see Sect. 4.2). These running conditions correspond to a maximum average luminosity of $1.5 \times 10^{31} /\left(\mathrm{cm}^{2} \mathrm{~s}\right)$. The ongoing upgrade of the HADES DAQ will enable operation up to $200 \mathrm{kHz}$. The increased DAQ rate combined with a more restrictive first level trigger will enable operation with a higher luminosity for the same maximum beam current by replacing the $\mathrm{LH}_{2}$ target with a polyethylene (PE) target of the same length. Taking the increased density of protons in the PE target $(\times 2)$ plus the protons in the carbon into account, the maximal luminosity for the PE target is expected to be up to a factor of 7 higher than for the $\mathrm{LH}_{2}$ target. This expectation is corroborated by our results obtained from pion induced reactions, where dilepton production from the PE target is very well described by the sum of pion-proton and quasifree pion-proton scattering $[39,40]$.

\subsection{Straw tracking stations}

The Straw Tracking Stations are based on self-supporting straw tube detectors with $10 \mathrm{~mm}$ inner diameter, which were built based on the design of the Forward Tracker (FT) and the central Straw Tube Tracker (STT) of the PANDA experiment $[37,41,42]$. The cathode of each straw consists of an aluminized Mylar foil with a thickness of $27 \mu \mathrm{m}$, and the anode is a gold-plated tungsten-rhenium wire with $20 \mu \mathrm{m}$ diameter. The operating pressure of the gas mixture is chosen to be 1 bar above the surrounding environment. This overpressure provides the necessary mechanical stiffness of the straws and makes them self-supporting without the need for massive support structure. The role of the detector frames is limited to the positioning of the straws.

Groups of 32 straw tubes are glued together in two staggered layers to form a module. The modules are mounted next to each other on a common support frame, thereby forming a double-layer of straw tubes. The design of the modules is 


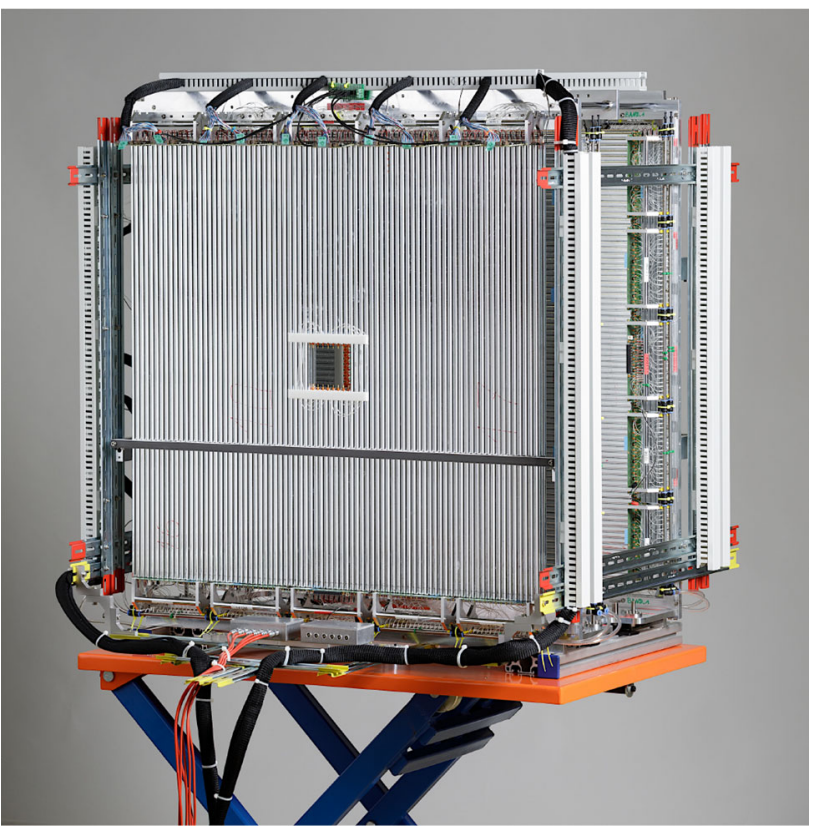

Fig. 2 The STS1 prepared for installation in HADES. (Photo: courtesy of Forschungszentrum Jülich $\mathrm{GmbH}$.)

described in Ref. [38] and allows for fast and simple maintenance by replacing single modules if necessary.

The STS1 and STS2 each consists of four double-layers. Groups of two double-layers are mounted on an individual support structure with one double-layer on each side of the support. The double-layers of straws are aligned by an inclination of $0^{\circ}, 90^{\circ}, 90^{\circ}$ and $0^{\circ}$ with respect to the vertical direction in consecutive double-layers of the STS1 (see Fig. 2). In the STS2 the straws in the first two double-layers are inclined by $90^{\circ}$ and $0^{\circ}$ and the next double-layers are inclined by $+45^{\circ}$ and $-45^{\circ}$ (see Fig. 3). This configuration facilitates an unambiguous reconstruction of multi-track events. The straws in the STS1 and STS2 have a length of $76 \mathrm{~cm}$ and $125 \mathrm{~cm}$, respectively. The double-layers are $80 \mathrm{~cm}$ and $112 \mathrm{~cm}$ wide, respectively. The double-layers in STS1 and STS 2 contain a central opening for the beam of $8 \times 8 \mathrm{~cm}$ and $16 \times 16 \mathrm{~cm}$, respectively. The opening is created by replacing the most central straws in the double-layers with pairs of shorter straws, leaving a gap between them. In total, the STS1 and STS2 stations contain 704 and 1024 individual straw tubes, respectively.

The pulses from the anode wires are amplified and discriminated in front-end electronic cards based on the PASTTREC ASIC [43]. Both the leading-edge time and the timeover-threshold of the detector signals are measured in multichannel TDCs implemented in the Trigger Readout Board version 3 (TRB3) [44].

The straw tubes are operated with a gas mixture of $\mathrm{ArCO}_{2}$ (90:10) at 2 bar absolute pressure. The operating voltage of the anode wires is $1800 \mathrm{~V}$, resulting in a gas gain of about

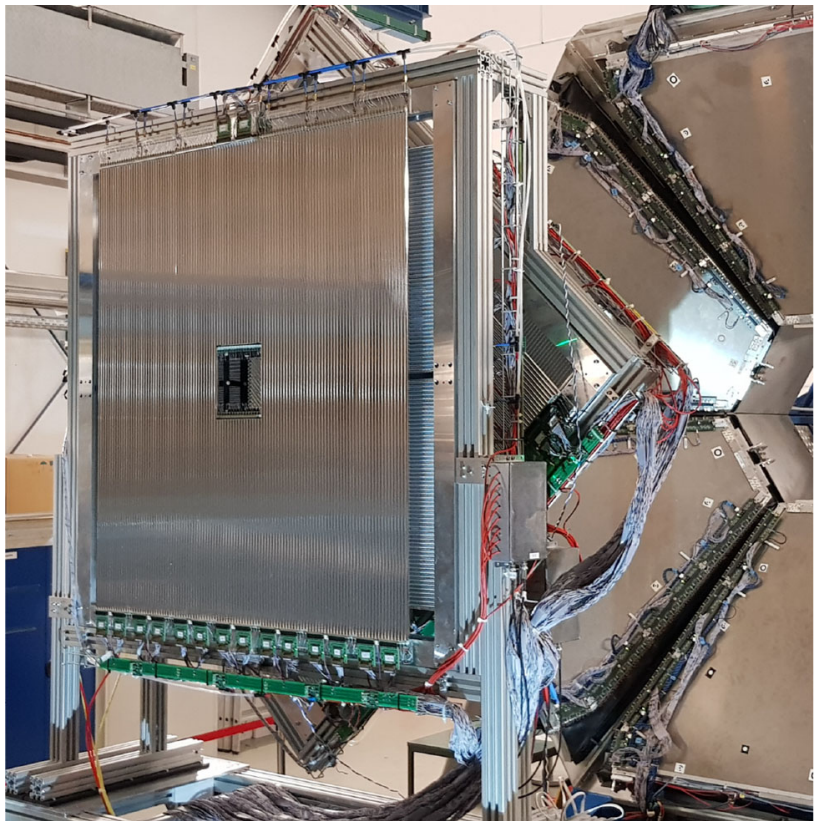

Fig. 3 STS2 installed in HADES in the maintenance position. The rear planes are rotated by $45^{\circ}$ around the beam axis with respect to the front one. The equipment behind the STS2 is part of the ECAL frame with the RPC detectors in front of it

$5 \times 10^{4}$ and a maximum drift time of about $130 \mathrm{~ns}$ for a maximum drift length equal to the straw tube radius $(5 \mathrm{~mm})$. The spatial resolution for each straw tube layer has been measured to be about $0.13 \mathrm{~mm}(\sigma)$ for minimum ionizing protons [38]. The average hit detection efficiency for protons with momentum of $2 \mathrm{GeV} / c$ incident on a single straw is measured to be over $95 \%$. The measured time-over-threshold of the detector signals is used to reject noise pulses and can also be used to determine the specific energy loss in order to distinguish protons, charged pions and kaons with momentum up to about $0.8 \mathrm{GeV} / c[45,46]$.

\subsection{The FRPC time-of-flight detector}

The FRPC is a ToF system in the Forward Detector based on individually shielded resistive plate chambers [47] and follows the existing concept in the HADES ToF wall [48], see Fig 1.

The FRPC detector consists of individually shielded hybrid (metal glass) strip-like RPC counters, which consist of three aluminum electrodes ( $2 \mathrm{~mm}$ thick) and two glass electrodes (1 mm thick), see Fig. 4a). The four gaps are defined by PEEK (Polyetheretherketone) mono-filaments that have $270 \mu \mathrm{m}$ diameter and are spaced every $90 \mathrm{~mm}$ along the counter. The assembly is housed inside individual shielding tubes and compressed with controlled force by springs. The springs act on the Polyvinyl chloride (PVC) plate, which distributes the force. High-voltage (HV) is applied to the central 

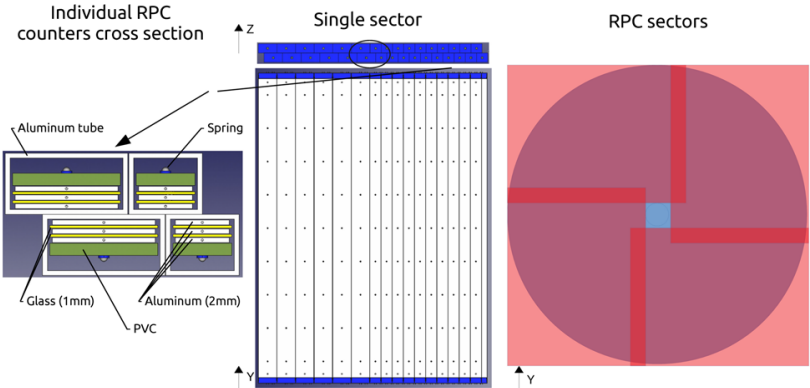

(a)

(b) $x$

(c)

Fig. 4 Drawing of the FRPC. (a) Cross sectional view of two $22 \mathrm{~mm}$ and two $42 \mathrm{~mm}$ width individual RPC counters, (b) single sector with two partially overlapping layers consisting of sixteen individual RPC counters each and (c) arrangement of the four RPC sectors

aluminum electrode, while the outer electrodes are grounded and the glass electrodes are kept electrically floating. Insulation to the shielding tube walls is assured by a double-layer KAPTON $^{\mathrm{TM}}$ adhesive laminate. An end-shield made of aluminum foil is spot-welded on the shielding tubes. The signals are collected in the central electrode, at both ends, through a $2 \mathrm{nF}$ HV coupling capacitor and transported outside the gas box through $50 \Omega$ cables and MMCX feedthroughs, that cross the lateral wall of the gas box. Signals are amplified by low-jitter high-gain/bandwidth Front-End Electronics (FEE) [49] and the ToF as well as the time-over-threshold of the detector signals is measured using the TRB3.

The counters are arranged in sectors, each with two partially overlapping layers, (see Fig. 4b). Each layer is composed of sixteen counters $750 \mathrm{~mm}$ long and two different widths of $22 \mathrm{~mm}$ to $42 \mathrm{~mm}$ in order to accommodate the spatial variation of the particle flux. The complete ToF detector is composed of four sectors arranged as shown in Fig. 4c), providing a hole of $130 \mathrm{~mm}$ diameter for the beam to pass through. The whole device is located at $7.5 \mathrm{~m}$ downstream of the target.

The ToF detector for the Forward Detector must be capable of measuring particle rates of $320 \mathrm{~Hz} / \mathrm{cm}^{2}$ with an efficiency better than $90 \%$ and a time resolution better than $100 \mathrm{ps}$. A test of a first prototype has been performed at the COSY accelerator with a beam of $2.2 \mathrm{GeV} / c$ protons. The obtained results show that the detector is able to fulfill these specifications for particle fluxes up to $250 \mathrm{~Hz} / \mathrm{cm}^{2}$ at room temperature. The glass resistivity drops by a factor 10 for an increase of the temperature by $25^{\circ} \mathrm{C}$, enabling a corresponding increase of the FRPC rate capability [50] by increasing the operational temperature of the detector. A rate of $500 \mathrm{~Hz} / \mathrm{cm}^{2}$ was obtained at $40^{\circ} \mathrm{C}$, with similar efficiency and resolution. A sustained rate of $1 \mathrm{kHz} / \mathrm{cm}^{2}$ was obtained with a modest drop of the detection efficiency down to $89 \%$.

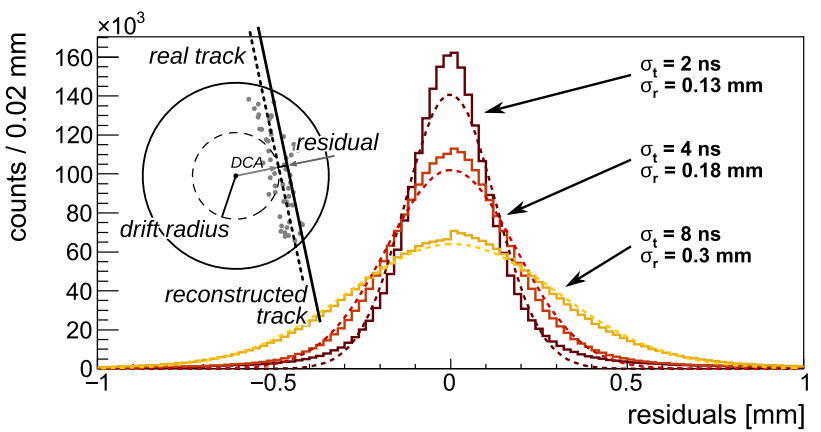

Fig. 5 Influence of the drift time smearing chosen in the simulations on the calculated track residual. The solid color distributions corresponds to residuals for different time resolutions, respective dotted lines are gaussian fits. (inlet) Charged particle passing the straws create electronpositive ion pairs. Electrons drift towards the anode and the drift radius is calculated from the drift time. The radius represents the distance of closest approach (DCA) between the particle track and the anode. The difference between the calculated drift radius and the reconstructed track is called the residual

In view of the need to increase the operating temperature of the detector to achieve (or exceed) the count rate requirements, the whole sector is made out of heat-resistant materials, chosen to withstand temperatures up to $65^{\circ} \mathrm{C}$, without loss of the relevant properties, and is equipped with a heating system.

\subsection{Digitization and track reconstruction}

Each individual straw is implemented in the HADES simulation framework taking into account the size and material of the straw film, gas volume and the anode wire. In the GEANT3 [51] based simulations the particle entrance and exit points of the tube are registered in order to approximate the track passing through the tube. For each tube with a registered hit, the digitization consists of first determining the track path by interpolating between the entrance and exit points with a line segment and then calculating the minimum distance to the center of the tube (i.e. neglecting the wire thickness) (see Fig. 5-inlet in the left). Then the trackto-wire distance is converted into the corresponding drift time by inverting the measured drift time spectrum [52]. In order to account for effects such as the time resolution of the front-end electronics, possible gain variations, temperature and systematic errors in the detector geometry, the drift time is convoluted with a Gaussian distribution with $\sigma=4 \mathrm{~ns}$ [46]. The finite detector inefficiency is accounted for by only storing the hit with $95 \%$ probability.

The track reconstruction proceeds in two steps: First a low-resolution (LR) tracking procedure is applied to the data. In this process only the straw anode coordinates are used, thereby limiting the tracking precision to the pitch of the straw detector anodes. Then the track is matched to a hit 


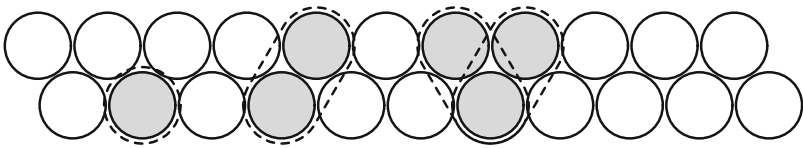

Fig. 6 Each circle represents an individual straw, and the fired straws are marked by gray filled circles. The dashed outlines show four possible types of clusters

in the FRPC. The timing signal from the FRPC is used to calculate the track-to-wire distances from the drift time in the individual straw detectors, thereby enabling a high-resolution (HR) tracking mode.

In the tracking algorithm, all registered hits from the same straw double-layer are grouped into clusters of one or two hits (neighboring channels, see Fig. 6). Successful tracking requires that there is a cluster in each double-layer of straws, thus the detection probability in a double-layer is $P=1-0.05^{2}=0.9975$. All combinations of clusters from all layers are fit with straight lines. Wrong combinations are rejected based on the $\chi^{2}$ value determined assuming a spatial resolution of $\sigma=3 \mathrm{~mm} \approx 10 \mathrm{~mm} / \sqrt{12}$. Only the best track candidates proceed through to the further tracking steps.

Two track fitting methods have been tested for the Forward Detector: (a) a Least Squares Method (LSM) adopted from the CBM-MUCH detector [53], and (b) a $\chi^{2}$ minimization adopted from the COSY-TOF detector [54]. The LSM uses an equation solving computation, whereas the $\chi^{2}$ minimization uses Minuit. The $\chi^{2}$ minimization method offers a more general solution, because LSM does not allow a track to be perpendicular to the beam axis (this case however does not allow hits in multiple double-layers due to the STS detector geometry). Track candidates reconstructed by the HR procedure are selected based on the $\chi^{2}$ value using a tracking resolution of $\sigma=200 \mu \mathrm{m}$ for each layer. In the final stage, tracks are sorted by their $\chi^{2}$ value from lowest to highest. Each pair of tracks which share at least half of their straws is considered and the track with the larger $\chi^{2}$ value is rejected. The track momentum is calculated using the track length and the FRPC ToF and assuming that the particle is a proton. In this case, the relative proton momentum resolution varies nearly linearly from $\delta p / p(\mathrm{FWHM})=0.5 \%$ at $0.5 \mathrm{GeV} / c$ to $3 \%$ at $2 \mathrm{GeV} / c$. Extrapolation of single tracks from the Forward Detector back to the target passes within $2.3 \mathrm{~mm}(\sigma)$ of the true vertex. The combination of two tracks to a hyperon decay vertex is measured with a precision of $\sigma_{x, y}=3 \mathrm{~mm}$, $\sigma_{z}=11.2 \mathrm{~mm}$ if one track is in the Forward Detector. If both tracks are in HADES the vertex resolution is $\sigma_{x, y}=4.2 \mathrm{~mm}$, $\sigma_{z}=9.4 \mathrm{~mm}$. The resolution of both the single track and the hyperon vertex is primarily limited by straggling in the air between the target and the Forward Detector, and does not significantly depend upon the drift time resolution of an individual straw, within the range $2 \mathrm{~ns}, 4 \mathrm{~ns}$ and $8 \mathrm{~ns}$ (Fig. 5).
Table 1 Production cross-section for the signal channels used in the benchmark simulations. See Section 3.1 for details

\begin{tabular}{lcc}
\hline Channel & $\sigma(\mu \mathrm{b})$ & Reaction Group \\
\hline$\Sigma(1385)^{0} \mathrm{X}$ & 56.2 & $\mathrm{AB}$ \\
$\Lambda(1405) \mathrm{X}$ & 32.2 & $\mathrm{AB}$ \\
$\Lambda(1520) \mathrm{X}$ & 69.6 & $\mathrm{AB}$ \\
$\mathrm{pK}^{+} \mathrm{K}^{+} \Xi^{-}$ & $0.35-3.6$ & $\mathrm{C}$ \\
$\Lambda \Lambda \mathrm{K}^{+} \mathrm{K}^{+}$ & $0.35-3.6$ & $\mathrm{D}$ \\
\hline
\end{tabular}

Results from tests of the straw detector exposed to a beam of $2 \mathrm{GeV} / c$ protons from the COSY accelerator at FZ-Jülich were compared to simulations. A test setup consisting of 16 double-layers arranged according to the PANDA Forward Tracker geometry: eight straw double-layers were inclined at $0^{\circ},+5^{\circ},-5^{\circ}, 0^{\circ}, 0^{\circ},+5^{\circ},-5^{\circ}$ and $0^{\circ}$, respectively and the distance between the layers also matched the Forward Tracker. The depth of the tracking stack was $70 \mathrm{~cm}$ compared to $183 \mathrm{~cm}$ for the Forward Detector. The custom tracking method used to analyze the data requires the first and the final tracking plane to always have double hit clusters. For a given pair of clusters, a corridor with a width of five straws is defined, and the cluster in each inner tracking layer is required to be within the corridor. The statistics of the number of fired straws in the inner layers (maximum 12 straws out of 16) follows the binomial distribution and yields a single straw efficiency of about $95 \%$ to $97 \%$ at $1750 \mathrm{~V}$. The applied tracking procedure considered only eight double-layers with vertical straws $0^{\circ}$ layers, and the remaining eight inclined layers were not used in order to more closely resemble the conditions of the Forward Detector. For the distribution of residuals, a standard deviation of $156 \mu \mathrm{m}$ was obtained, which is consistent with the assumed drift time smearing of $4 \mathrm{~ns}$.

\section{Benchmark channels}

Several groups of benchmark channels have been chosen in order to investigate the performance of the upgraded HADES detector to measure the following processes in proton-proton collisions at a beam kinetic energy of $E_{\text {kin }}=4.5 \mathrm{GeV}$ :

(A) real photon electromagnetic decay of hyperons,

(B) virtual photon electromagnetic decay of hyperons,

(C) double strangeness $\left(\Xi^{-}\right)$production,

(D) double $\Lambda$ production and their correlations.

A full list of the production cross-sections for the signal channels studied in this work is provided in Table 1. The corresponding decay branching ratios are listed in Table 2 . Except for the channels with real photon decay, the signal reconstruction strategy assumes a semi-inclusive reconstruc- 


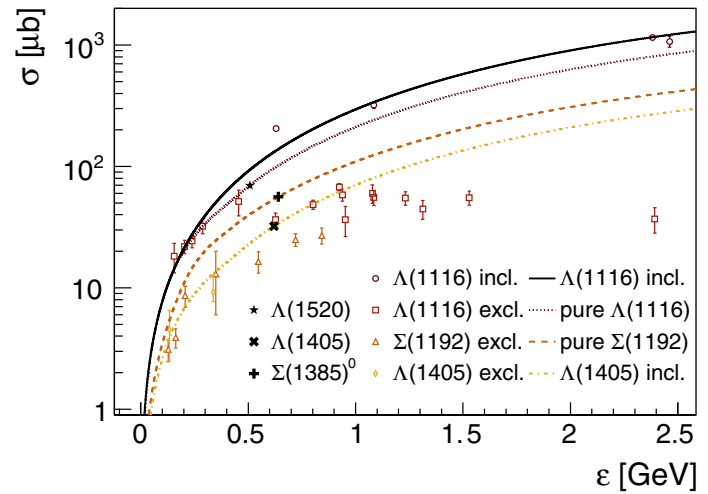

Fig. 7 Hyperon production cross-section as a function of excess energy $\epsilon$. The solid black line represents a parameterization of $\sigma_{\mathrm{pp} \rightarrow \Lambda \mathrm{X}}$ (see text for details). The dotted and dashed lines represent a decomposition of the inclusive $\Lambda$ cross-section into $\Lambda$ corrected for the $\Sigma^{0}$ feed-down (dotted) and $\Sigma^{0}$ (dashed) contributions. The short-dashed line is a parameterization of $\sigma_{\mathrm{pp} \rightarrow \Lambda(1405) \mathrm{X}}$, obtained as $1 / 3$ of $\sigma_{\mathrm{pp} \rightarrow \Lambda \mathrm{X}}$ (see text for details). The black full symbols represent the estimated inclusive cross-sections for $\Lambda(1520), \Lambda(1405)$ and $\Sigma(1385)^{0}$ for ppreactions with $E_{\text {kin }}=4.5 \mathrm{GeV}$. The open symbols represent existing experimental data

tion tagged by the $\Lambda \rightarrow \mathrm{p} \pi^{-}$weak decay with hyperons reconstructed using the invariant mass of their decay products. This strategy takes advantage of both the larger acceptance for inclusive reconstruction in HADES and the larger inclusive cross-sections. However, the real photon decay of hyperons requires exclusive reconstruction in order to reject background stemming from neutral pion decays. The dominant background channels included in the simulations are listed in Table 3. They can be grouped into reactions with final states including: (a) at least one proton and multi-pions (with at least one negative charge) (b) associated strangeness production with $\Lambda / \Sigma^{0}$ hyperons, and (c) non-strange resonances e.g. $\Delta(1232)$, relevant for the studies of Dalitz-decays.

\subsection{Cross-section estimates}

No data on the inclusive production cross-sections of the hyperon resonances $\Sigma(1385)^{0}, \Lambda(1405), \Lambda(1520)$ (denoted in the article as $\mathrm{Y}$ ) and $\Xi^{-}$hyperons exist for proton-proton reactions in the energy region of interest. Thus, the following cross-section estimates are based on the available data on $\Lambda / \Sigma^{0}$ production.

\subsubsection{Production cross-sections of excited hyperons}

Inclusive production of hyperon resonances (see Table 1) is approximated in simulation by the most dominant reaction channel of the type $\mathrm{pK}^{+} \mathrm{Y}$ with cross-sections calculated as explained below.

Cross-sections for the exclusive reaction $\mathrm{pp} \rightarrow \mathrm{pK}^{+} \Lambda$ have been measured in the range $2.5 \mathrm{GeV}<\sqrt{s}<6 \mathrm{GeV}$ by sev- eral experiments: COSY-TOF [55], HADES [56] and older ones summarized in [57] (see Fig. 7). Data on the inclusive cross-section for $\mathrm{pp} \rightarrow \Lambda$ Xare sparse; there are only four data points in the energy range of interest. The inclusive $\Lambda$ excitation function is described by using a third order polynomial in order to interpolate these data points and the exclusive data for energies close to the production threshold. The interpolation function, denoted by the solid line is:

$\sigma_{\mathrm{pp} \rightarrow \Lambda \mathrm{X}}=47.97 \epsilon+292.6 \epsilon^{2}-45.36 \epsilon^{3} \mu \mathrm{b}$

with the excess energy $\epsilon=\sqrt{s}-\sqrt{s_{\mathrm{th}}}$, where $\sqrt{s_{\mathrm{th}}}=$ $2.55 \mathrm{GeV}$ is the threshold for $\Lambda$ production.

Since the branching ratio for $\Sigma^{0}$ into $\Lambda \gamma$ is almost $100 \%$, the inclusive $\Lambda$ cross-section includes the feed-down from $\Sigma^{0}$ production. Nevertheless, it is possible to disentangle these two channels using the measured $\Lambda / \Sigma^{0}$ ratio in exclusive channels, as given in [58]. Thus, the measured $\Lambda / \Sigma^{0}$ cross-section ratio was parameterized by the COSY parameterization [55] for $\epsilon<0.275 \mathrm{GeV}$ and a linear function

$\frac{\sigma_{\Lambda \mathrm{X}}}{\sigma_{\Sigma^{0} \mathrm{X}}}(\epsilon)=2.215-0.027 \epsilon$

to describe the ratio for $\epsilon>0.275 \mathrm{GeV}$. The parameterization of the inclusive $\Lambda$ production cross-section, corrected for $\Sigma^{0}$ feed-down, is shown in Fig. 7 by the dotted line. The corresponding parameterization for the $\Sigma^{0}$ is plotted with a dashed line.

The cross-section for $\mathrm{pp} \rightarrow \Lambda(1520) \mathrm{X}$ is estimated assuming the same matrix element as for the $\mathrm{pp} \rightarrow \Lambda \mathrm{X}$ reaction, after correction for the $\Sigma^{0}$ feed-down. Consequently, it was assumed that the only difference between the crosssections for these two channels at a given $\sqrt{s}$ is the volume of available phase-space. The respective threshold energy to produce the $\Lambda(1520)$ is $\sqrt{s_{\mathrm{thr}}}=2.95 \mathrm{GeV}$, thus at $E_{\mathrm{kin}}=$ $4.5 \mathrm{GeV}(\sqrt{s}=3.46 \mathrm{GeV})$ the excess energy corresponds to $\epsilon=0.51 \mathrm{GeV}$. At this $\epsilon$ and assuming

$\sigma_{\Lambda \mathrm{X}}(\sqrt{s}=2.55+\epsilon) \approx \sigma_{\Lambda(1520) \mathrm{X}}(\sqrt{s}=2.95+\epsilon)$

a value of $\sigma_{\Lambda(1520) \mathrm{X}}=69.6 \mu \mathrm{b}$ is obtained.

The cross-section for inclusive $\Sigma(1385)^{0}$ production was calculated in a similar way as for the $\Lambda(1520)$. The input parameterization obtained for the $\Sigma^{0}$ was used under the assumption that the difference between the $\Sigma(1385)$ and the $\Sigma^{0}$ production at the given $\sqrt{s}$ is governed only by the volume of available phase-space. The respective crosssection for the inclusive production at $E_{\text {kin }}=4.5 \mathrm{GeV}$ has been estimated in this way to be $\sigma_{\Sigma(1385) \mathrm{X}}=56.5 \mu \mathrm{b}$.

The cross-section for the exclusive $\Lambda(1405)$ production was measured by ANKE [59] and HADES [60]. An empirical relation between the cross-section as a function of the excess energy for $\Lambda(1405)$ and $\Lambda$ was deduced from these data to be: $\frac{1}{3} \sigma_{\Lambda \mathrm{K}^{+} \mathrm{p}}(\epsilon)=\sigma_{\Lambda(1405) \mathrm{K}^{+} \mathrm{p}}(\epsilon)$ in [60]. Using this 
Table 2 List of branching ratios for the signal and background channels, see Sect. 3.1 for details. The "Reaction Group" column denotes the reaction from Table 1 for which this BR is used

\begin{tabular}{lcc}
\hline Channel & Branching Ratio & Reaction Group \\
& & \\
\hline$\Sigma(1385)^{0} \rightarrow \Lambda \gamma$ & $1.4 \times 10^{-2}$ & $\mathrm{~A}$ \\
$\Sigma(1385)^{0} \rightarrow \Lambda \mathrm{e}^{+} \mathrm{e}^{-}$ & $1.4 \times 10^{-4}$ & $\mathrm{~B}$ \\
$\Lambda(1405) \rightarrow \Lambda \gamma$ & $5.0 \times 10^{-4}$ & $\mathrm{~A}$ \\
$\Lambda(1405) \rightarrow \Lambda \mathrm{e}^{+} \mathrm{e}^{-}$ & $4.9 \times 10^{-6}$ & $\mathrm{~B}$ \\
$\Lambda(1520) \rightarrow \Lambda \gamma$ & $1.1 \times 10^{-2}$ & $\mathrm{~A}$ \\
$\Lambda(1520) \rightarrow \Lambda \mathrm{e}^{+} \mathrm{e}^{-}$ & $1.1 \times 10^{-4}$ & $\mathrm{~B}$ \\
$\Delta^{+} \rightarrow \mathrm{pe}^{+} \mathrm{e}^{-}$ & $4.5 \times 10^{-5}$ & $\mathrm{~B}$ \\
$\Delta^{0} \rightarrow \mathrm{ne}^{+} \mathrm{e}^{-}$ & $4.5 \times 10^{-5}$ & $\mathrm{~B}$ \\
\hline
\end{tabular}

relation and the parameterization for inclusive $\Lambda$ production, the inclusive cross-section for $\Lambda(1405)$ production is estimated to be $32 \mu \mathrm{b}$ at $\sqrt{s}=3.46 \mathrm{GeV}$. The results are summarized in Table 1.

\subsubsection{Decay branching ratios of excited hyperons}

The branching ratios (BR) for the real photon radiative transition of $\Sigma(1385)^{0}, \Lambda(1520)$ were obtained from CLAS $[15,16]$ and are given in Table 2 . The branching ratios for the Dalitz-decay of these states have been estimated from the measured branching ratio for real photon decay using the following formula [22]:

$\mathrm{d} \Gamma^{\mathrm{Y} \rightarrow \Lambda \mathrm{e}^{+} \mathrm{e}^{-}} M_{\mathrm{ee}} \simeq \frac{2 \alpha}{3 \pi M_{\mathrm{ee}}} \Gamma^{\mathrm{Y} \rightarrow \Lambda \gamma}$,

where $\alpha$ is the fine structure constant and $M_{\mathrm{ee}}$ is the dilepton invariant mass. After integration over $M_{\mathrm{ee}}$ this simplifies to

$\Gamma^{\mathrm{Y} \rightarrow \Lambda \mathrm{e}^{+} \mathrm{e}^{-}}=1.35 \cdot \alpha \Gamma^{\mathrm{Y} \rightarrow \Lambda \gamma}$.

\subsubsection{Production of double strangeness}

The cross-section for $\mathrm{pp} \rightarrow \mathrm{pK}^{+} \mathrm{K}^{+} \Xi^{-}$at $\sqrt{s}=3.46 \mathrm{GeV}$ $(\epsilon=0.2 \mathrm{GeV})$ has been estimated from the cross-section ratio $\sigma_{\Xi^{-} \mathrm{X}} / \sigma_{\left(\Lambda+\Sigma^{0}\right) \mathrm{X}}=[1.2 \pm 0.3$ (stat) \pm 0.4 (syst) $] \times 10^{-2}$ measured in proton-nucleus interactions at $E_{\text {kin }}=3.5 \mathrm{GeV}$ by HADES [27]. The cross-section for inclusive $\Lambda$ production in proton-proton collisions (uncorrected for the $\Sigma^{0}$ feed-down) has been parameterized as described above. At $E_{\text {kin }}=4.5 \mathrm{GeV}$ the $\Xi^{-}$production cross-section is thus estimated to be $3.56 \mu \mathrm{b}$.

This estimate is based on the measured ratio $\sigma_{\Xi^{-} \mathrm{x}} /$ $\sigma_{\left(\Lambda+\Sigma^{0}\right) \mathrm{X}}$ in proton-nucleus interactions, which might be strongly affected by medium effects. In fact, the measured ratio is surprisingly large and exceeds predictions from the
UrQMD transport model [27]. Possible explanations of this puzzling result are given in [28], including decays of higher mass non-strange resonances with sizeable branching ratios into $\Xi \mathrm{KK}$. If such a mechanism is significant, it should also be active in proton-proton interactions. Hence, this estimate is considered as an upper limit for the production crosssection. In order to make an alternative estimate, the crosssection ratio $\sigma_{\Xi^{-} \mathrm{X}} / \sigma_{\left(\Lambda+\Sigma^{0}\right) \mathrm{X}}$ at the same excess energy has been calculated from existing $\mathrm{p}+\mathrm{p}$ data. The lowest energy for which the cross-section of $\mathrm{pp} \rightarrow \Xi^{-} \mathrm{KKX}$ was measured ( $\sigma=7 \mu \mathrm{b}$ ) corresponds to $\sqrt{s}=4.54 \mathrm{GeV}$ or $\epsilon=1.29 \mathrm{GeV}$ [57]. At this excess energy the inclusive cross-section for $\Lambda$ production is $\sigma_{\Lambda \mathrm{X}}=451 \mu \mathrm{b}$ as estimated from Eq. (1). Using these data, the ratio $\sigma_{\Xi^{-} \mathrm{X}} / \sigma_{\left(\Lambda+\Sigma^{0}\right) \mathrm{X}}$ is 0.015 . Extrapolating this ratio to $E_{\mathrm{kin}}=4.5 \mathrm{GeV}$ results in a value of $\sigma_{\Xi^{-} \mathrm{X}}=0.35 \mu \mathrm{b}$, which is taken as an alternative estimate of the production cross-section in these simulations.

The exclusive production cross-section for $\Lambda \Lambda\left(\sqrt{s_{t h}}=\right.$ $3.21 \mathrm{GeV}$ ) was taken to be equal to the cross-section for $\mathrm{pp} \rightarrow$ $\mathrm{pK}^{+} \mathrm{K}^{+} \Xi^{-}\left(\sqrt{s_{t h}}=3.236 \mathrm{GeV}\right)$. This is motivated by the same strange quark content, meson number, and the minimal difference in the volume of available phase-space due to the different threshold energies of about $26 \mathrm{MeV}$.

\subsubsection{Background channels}

A list of the dominant background channels included in these simulations is presented in Table 3. These background channels contain the same final state particles as for the signal channels, e.g. $p \pi^{-} \mathrm{e}^{+} \mathrm{e}^{-}$for hyperon Dalitz-decays, $\mathrm{p} \pi^{-} \pi^{-}$ for $\Xi^{-}$, and $p p \pi^{-} \pi^{-}$for $\Lambda \Lambda$.

Background contributions to real photon hyperon decays (denoted by A in Table 3 ) were selected to contain $\mathrm{pK}^{+} \Lambda$ in the final state (channels 6-11). Additional multipion channels (1 and 5) account for $\pi^{+}$misidentification as $\mathrm{K}^{+}$.

The main background contributions to hyperon Dalitzdecays (denoted by $\mathrm{B}$ in Table 3 ) originates from channels containing $\Lambda$ and a dilepton source (mainly $\pi^{0}$ Dalitzdecays, channels 9 and 11). An important source of background in the higher dilepton invariant mass region (i.e. $\left.M_{\mathrm{e}^{+} \mathrm{e}^{-}}>M_{\pi^{0}}\right)$ is related to $\Delta$ Dalitz-decay, associated with $\Lambda$ production (channels 7 and 9 ). The background originating from Dalitz-decays of non-strange resonances without a $\Lambda$ in the final state but emitted together with $\mathrm{p} \pi^{-}$pair (demanded in the $\Lambda$ reconstruction) was estimated by contributions from $\Delta^{+, 0}$ because of their relatively large branching ratio to dileptons. Cross-sections for these channels were approximated by the corresponding data for multi-pion production, assuming that pions originate only from the $\Delta$ decays (channels 2 and 4). This approach is taken to be an upper limit for this background contribution.

Combinatorial background in the dielectron measurements is determined from Dalitz-decays and/or external con- 
Table 3 List of background channels used in the benchmark simulations. See Sect. 3.1 for details. The "Reaction Group" column denotes the reaction in Table 1 for which the given channel is used

\begin{tabular}{rlcc}
\hline & Channel & $\sigma(\mathrm{pb})$ & Reaction Group \\
\hline 1 & $\mathrm{pp} \pi^{+} \pi^{-} \pi^{0}$ & 1840 & $\mathrm{AB}$ \\
2 & $\mathrm{p} \pi^{+} \pi^{-} \Delta^{+}$ & 2760 & $\mathrm{~B}$ \\
3 & $\mathrm{pn} \pi^{+} \pi^{+} \pi^{-} \pi^{0}$ & 300 & $\mathrm{~B}$ \\
4 & $\mathrm{p} \pi^{+} \pi^{+} \pi^{-} \Delta^{0}$ & 450 & $\mathrm{~B}$ \\
5 & $\mathrm{pp} \pi^{+} \pi^{-} \pi^{0} \pi^{0}$ & 300 & $\mathrm{AB}$ \\
6 & $\mathrm{p} \Lambda \mathrm{K}^{+}$ & 54.5 & $\mathrm{~A}$ \\
7 & $\mathrm{p} \Lambda \mathrm{K}^{+} \pi^{+} \pi^{-}$ & 20 & $\mathrm{~A}$ \\
8 & $\mathrm{p} \Sigma^{0} \mathrm{~K}^{+}$ & 23.5 & $\mathrm{~A}$ \\
9 & $\mathrm{p} \Sigma^{0} \mathrm{~K}^{+} \pi^{0}$ & 20 & $\mathrm{~A}$ \\
10 & $\mathrm{p} \Sigma^{0} \mathrm{~K}^{+} \pi^{+} \pi^{-}$ & 2 & $\mathrm{~A}$ \\
11 & $\mathrm{p} \Lambda \mathrm{K}^{+} \pi^{0}$ & 43 & $\mathrm{AB}$ \\
12 & $\Delta^{+} \mathrm{K}^{+} \Lambda$ & 64 & $\mathrm{~B}$ \\
13 & $\mathrm{n} \Lambda \mathrm{K}^{+} \pi^{+} \pi^{0}$ & 20 & $\mathrm{~B}$ \\
14 & $\Delta^{0} \pi^{+} \mathrm{K}^{+} \Lambda$ & 30 & $\mathrm{~B}$ \\
15 & $\mathrm{p} \Lambda \mathrm{K}^{+} \pi^{0} \pi^{0}$ & 10 & $\mathrm{~B}$ \\
16 & $\mathrm{p} \Lambda \mathrm{K}^{+} \pi^{0} \pi^{0} \pi^{0}$ & 7 & $\mathrm{~B}$ \\
17 & $\mathrm{p} \Sigma^{0} \mathrm{~K}_{\mathrm{S}}^{0} \pi^{+}$ & 9 & $\mathrm{BC}$ \\
18 & $\mathrm{pp} \pi^{+} \pi^{+} \pi^{-} \pi^{-}$ & 227 & $\mathrm{CD}$ \\
19 & $\mathrm{p} \Lambda \mathrm{K}_{\mathrm{S}}^{0} \pi^{+}$ & 30 & $\mathrm{CD}$ \\
20 & $\mathrm{p} \Lambda \mathrm{K}^{+} \pi^{+} \pi^{-}$ & 21 & $\mathrm{CD}$ \\
21 & $\mathrm{n} \Lambda \mathrm{K}_{\mathrm{S}}^{0} \pi^{+} \pi^{+}$ & 10 & $\mathrm{C}$ \\
22 & $\mathrm{pp} \mathrm{K}_{\mathrm{S}}^{0} \mathrm{~K}_{\mathrm{S}}^{0}$ & 1.6 & $\mathrm{CD}$ \\
\hline
\end{tabular}

version of photons from neutral pions. Hence, production channels with one or multiple $\pi^{0}$ were also included in the simulations.

The most abundant background channels for the $\Xi^{-}$ (denoted by $\mathrm{C}$ in Table 3 ) are related to multi-pion production (channels 17 to 22), which have much larger production cross-sections than the corresponding signal channels. Fortunately, they can be effectively suppressed by applying a condition on the displaced $\mathrm{p} \pi^{-}$decay vertex of the $\Lambda$. Therefore, the important background channels contain a $\Lambda$ hyperon and at least one $\pi^{-}$meson. A special case for this class of reactions contains pions produced at displaced vertices w.r.t. the primary interaction vertex. This situation can occur when a $\Lambda$ is produced associated with a $\mathrm{K}_{\mathrm{S}}^{0}$ meson (channels 17,19 , 21 and 22). The $\mathrm{D}$ group related to the $\Lambda \Lambda$ production contains the same background channels as the remaining $\mathrm{p}$ and $\pi^{-}$in the final state can contribute to additional $\Lambda$.

Existing cross-section data for the background channels without strangeness were taken wherever possible from the compilation in Ref. [57]. If no data on a given channel are available at our beam energy, the results from higher energies were used, therefore the assumed cross-sections provide an upper estimate for the expected background. Crosssections for most of the strangeness-containing background channels were based on measurements of different channels at various beam energies collected from [61] and fit with a phase space distribution according to:

$\sigma(E)=a \cdot\left(1-\frac{E_{0}+2 m_{\mathrm{p}}}{E+2 m_{\mathrm{p}}}\right)^{b} \cdot\left(1-\frac{E_{0}}{E+2 m_{\mathrm{p}}}\right)^{c}$,

where $E$ is the kinetic energy of the beam particle, $E_{0}$ is the threshold beam energy for the production of the channel and $m_{\mathrm{p}}$ is the proton mass.

\section{Simulation and analysis techniques}

The selected benchmark and background channels have been simulated using the Pluto Monte-Carlo event generator [62] and processed with GEANT3 to model the detector acceptance. The simulations were performed for a proton beam with $4.5 \mathrm{GeV}$ incident on a $4.7 \mathrm{~cm}$ long $\mathrm{LH}_{2}$ target.

Simulated events were reconstructed with the same methods as for experimental data using the hydra software framework [48]. The effect of the trigger applied during data taking was studied using a dedicated emulator, as described below.

\subsection{Acceptance for hyperon decays}

The daughter baryon from hyperon decay will be strongly forward peaked in the laboratory reference frame as a consequence of three main effects: $(a)$ the produced hyperons have an anisotropic angular distribution in the center of momentum reference frame (c.m.) (b) the decay kinematics of the hyperons and $(c)$ the boost of the final state particles into the laboratory frame resulting from the fixed target kinematics. The kinematic boost is the most important of these three effects for the given set of reaction channels and beam energy.

The angular distribution in the c.m. reference frame of $\Lambda$ produced at a beam kinetic energy of $E_{\text {kin }}=3.5 \mathrm{GeV}$ shows about a factor two enhancement for forward emitted hyperons relative to $\theta_{c . m}$. $=90^{\circ}$ [56]. This asymmetry is however expected to be significantly smaller for the production of the heavier $\Sigma^{*} / \Lambda^{*}$ states, $\Xi^{-}$and $\Lambda \Lambda$ in this study, which are produced at smaller excess energies $\epsilon \sim 0.2 \mathrm{GeV}$ to $0.5 \mathrm{GeV}$, and thus is neglected below.

The daughters from the hyperon decays relevant for these studies include a proton and either one pion for $\Lambda \rightarrow \mathrm{p} \pi^{-}$or two pions for the cascade, which proceeds through two consecutive weak decays: $\Xi^{-} \rightarrow \Lambda \pi^{-}$and $\Lambda \rightarrow p \pi^{-}$. The large mass ratio between the proton and pion, together with the relatively small decay phase space for the hyperons result in the proton being emitted in a direction very close to the hyperon direction. In contrast, the pion(s) will be emitted over a much wider angular range relative to the direction of the hyperon. This effect is visible in Fig. 8 $(\mathrm{a}, \mathrm{b})$ for the pion and proton daughters of $\Lambda(1520)$ decay, respectively. Similarly, the pion and proton daughters from $\Xi^{-}$decay are shown in frames (c) 


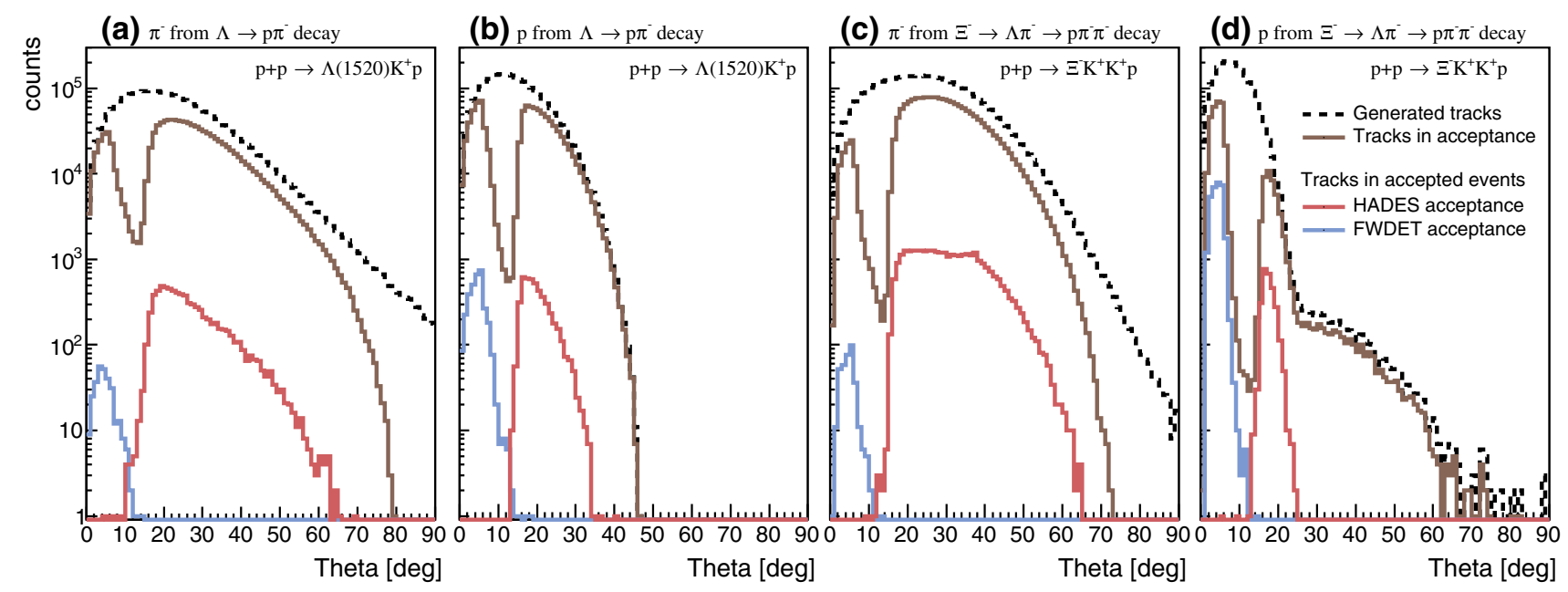

Fig. 8 Polar angle distribution of the (a) pions and (b) protons emitted from $\Lambda(1520)$ decay following the $\mathrm{pp} \rightarrow \mathrm{pK}^{+} \Lambda(1520)\left(\Lambda \gamma^{*}\right)$ reaction, and (c) pions and (d) protons emitted from $\Xi^{-}$decay following the $\mathrm{pp} \rightarrow \mathrm{pK}^{+} \mathrm{K}^{+} \Xi^{-}$reaction. The black dashed curve shows the distribution for all corresponding particles created in the collisions. The brown line shows the inclusive distribution for those emitted within either the HADES or the Forward Detector acceptance. The red and the blue lines correspond to particles in the HADES and Forward Detector acceptance, respectively, which are registered within fully reconstructed events i.e. all required tracks of the full event are within the acceptance of HADES or the Forward Detector and (d), respectively. The dashed black lines show the distributions of the particles emitted in the full solid angle, and the solid (brown) lines show the corresponding distribution for particles measured within the acceptance of either HADES or the new Forward Detector. Although most of the protons are emitted forward, a tail to larger angles is visible in frame (d), that results from decays after rescattering of the $\Xi^{-}$on the target or the detector materials. The characteristic dip around $10^{\circ}$ is due to the lack of acceptance in the region between the new Forward Detector and the existing HADES due to the magnet support ring. The blue and red solid curves in Fig. 8 show the corresponding distributions for accepted particles in the Forward Detector and HADES, respectively, for those events in which all final state particles are registered in the detector acceptance. The distributions clearly demonstrate that most pions are emitted into the HADES acceptance, whereas the protons are emitted preferentially into the forward direction with a large fraction detected in the Forward Detector.

The quantitative importance of the new Forward Detector for hyperon reconstruction close to the production threshold can be seen from the following results: $88 \%$ of events with all daughters of the $\Xi^{-}$inside the acceptance of HADES or the Forward Detector have both pion tracks in the HADES acceptance and the proton track in the Forward Detector. Only $11 \%$ of $\Lambda \Lambda$ events are completely inside the HADES acceptance and the remaining $89 \%$ include at least one particle in the Forward Detector. The Forward Detector detects $41 \%$ of all accepted protons tracks for the $\Lambda(1520)$ decays. The smaller acceptance gain for the $\Lambda(1520)$ by adding the
Forward Detector compared to the $\Xi^{-}$is a consequence of the higher excess energy for this reaction $(\epsilon=0.5 \mathrm{GeV})$. The increased acceptance by a factor of two due to the Forward Detector is sizeable and important for reconstruction of rare Dalitz-decays. These simulations can be taken as a lower estimate of the detector acceptance, because any additional forward peaking of the production differential crosssection in the c.m. frame will lead to a further increase of the Forward Detector acceptance.

\subsection{Trigger filtering}

An efficient and selective trigger condition is a key factor for the planned experiments. A fast trigger applied in HADES uses multiplicity information based on the number of hits in both of the time of flight META (Multiplicity Electron Trigger Array) detectors: TOF and RPC. For this work the same trigger conditions are applied that were used during the p+pexperiment at $3.5 \mathrm{GeV}$ investigated by HADES in 2007 . The multiplicity trigger used in that experiment was set to at least three hits in META (so-called "M3" trigger). The M3 trigger seems to be an optimal trigger for the planned measurements of hyperons because it strongly suppresses background from two-prong events and at the same time does not significantly reduce the number of reconstructed hyperon yield in the HADES acceptance.

Dalitz-decays of hyperon resonances $\mathrm{Y} \rightarrow \Lambda\left(\mathrm{p} \pi^{-}\right) \mathrm{e}^{+} \mathrm{e}^{-}$ produce four particles that need to be reconstructed. Simulation studies of $\Lambda(1520)$ decays show that the M3 trigger condition does not significantly reduce the number of 
reconstructed events inside the HADES acceptance. Figure 8 shows that either all four tracks are observed in HADES $(59 \%)$ or three in HADES and one (proton) in the Forward Detector $(41 \%)$.

Reconstruction of $\Xi^{-}$decay requires the proton from the secondary $\Lambda$ decay to be detected in the Forward Detector and both pions to be detected in HADES. This situation occurs with $88 \%$ probability, thus, one of the associated kaons must also be detected in HADES in order to fulfill the M3 condition. Note that most spectator protons are detected in the Forward Detector, similar to protons from $\Lambda$ decay. The simulations show that the M3 trigger condition reduces the number of reconstructed $\Xi^{-}$events by only $10 \%$.

For the $\Lambda \Lambda$ reconstruction both pions from $\Lambda$ decays are emitted into HADES and the two protons either both enter the Forward Detector or one of them enters the Forward Detector and the second goes through HADES. In this case the M3 trigger reduces the number of reconstructed $\Lambda \Lambda$ events by only $3 \%$, as most of the time at least three particles hit the HADES.

\subsection{Track reconstruction and particle identification}

The particle tracking in HADES is based on the four planes of the multi-wire drift chambers (MDC) with each plane consisting of 6 wire layers. Two planes are located in front of the toroidal magnetic field and the other two behind the field, allowing the track deflection to be measured (see Fig. 1). Each pair of planes is positioned in a magnetic field-free region, where straight track segments are reconstructed. The track segments before and after the magnetic field volume are connected by a curved track section. The momentum of the particle is calculated from the curvature of the track in the magnetic field (see [1] for details). Next, the reconstructed track is matched with an associated hit in the TOF or RPC detectors. The reconstructed path length and the ToF information are combined to calculate the particle velocity $(\beta c)$ and conditions on the correlation between the momentum and the velocity can be used to differentiate between particle species of different mass. Depending on the simulated channel, the particle identification (PID) methods applied slightly differ. For the $\Xi^{-}$and $\Lambda \Lambda$ analyses, the PID selection is based on selection bands calculated by varying the expected velocity (expressed as $\beta_{\mathrm{c}}$ ) for a given mass hypothesis and momentum, as shown by the solid lines in Fig 9. For the hyperon Dalitz-decays, the PID is determined by calculating the mass squared from the measured $\beta$ and momentum. Particles with squared masses in the range $40 \mathrm{MeV} / c^{2}$ to $240 \mathrm{MeV} / c^{2}$ and $650 \mathrm{MeV} / c^{2} 1127 \mathrm{MeV} / c^{2}$ are used to identify $\pi$ and $\mathrm{p}$, respectively. Leptons are identified by matching the reconstructed track with a spatially correlated ring measured in the RICH (for details see [1]). For the reconstruction of real

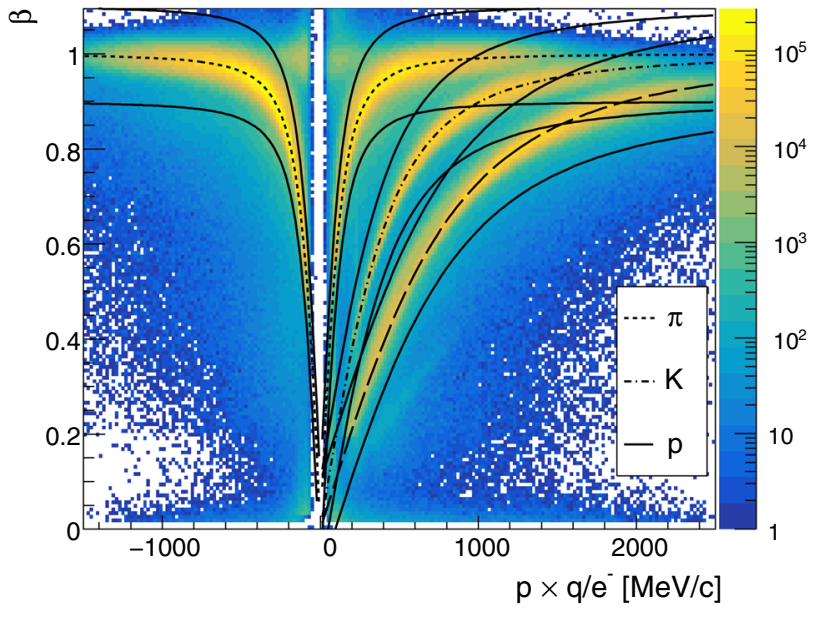

Fig. 9 Particle identification based on the $\beta$ vs momentum correlation measured in HADES. The dashed, dot-dashed and dotted lines designate the $\beta_{c}$ reference for protons, kaons and pions, respectively. The solid lines denote the $\beta_{\mathrm{c}} \pm 0.075$ selection range for each particle species

photon decay of hyperons, the PID was based on a neural network, as described in Sect. 5.1.

Unlike the HADES detector, the Forward Detector is positioned entirely in a region with no magnetic field, thus no direct momentum measurement is possible. Instead, for a given mass hypothesis the momentum of a track can be deduced by combining the flight path information of the Forward Detector with the ToF measurement in the FRPC. Although the measured ToF is not sufficient to completely separate protons from pions and kaons by itself (see Fig. 10), it is nevertheless sufficient to provide an important constraint for various event hypotheses, since the path lengths have very little dispersion. For example, by demanding that ToF $>27 \mathrm{~ns}$, as indicated by the dashed line in Fig. 10, the pion contribution is suppressed by $45 \%$ compared to only $0.7 \%$ for the proton yield.

These studies show that protons from hyperon decays are emitted preferentially in the forward direction and the Forward Detector plays an important role in their detection. Hence, for the $\Xi^{-}$and $\Lambda \Lambda$ reconstruction the event hypothesis requires that all particles in the Forward Detector are protons, and that both pions are in the HADES acceptance and identified as pions based on the PID methods explained above. For the hyperon Dalitz-decays, a pair of leptons and a pion must be found in HADES, and the remaining daughter proton is either identified in HADES or reconstructed in the Forward Detector.

\subsection{Inclusive $\Lambda$ reconstruction}

Reconstruction of the $\Lambda$ is common to all analyses described here. As a neutral particle with the dominant $\mathrm{p} \pi^{-}$weak 


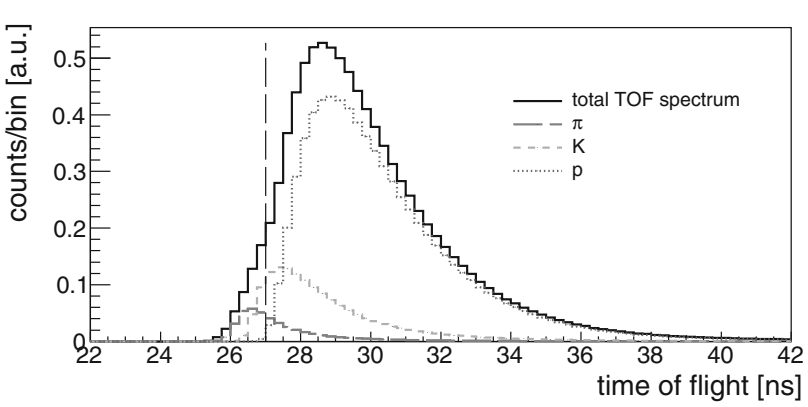

Fig. 10 ToF spectra for different particle species measured in the FRPC, produced in the reaction $\mathrm{pp} \rightarrow \mathrm{pK}^{+} \mathrm{K}^{+} \Xi^{-}$

decay it can be identified by its displaced pion-proton vertex $(c \tau=7.89 \mathrm{~cm})$. As discussed in the previous section, the event hypothesis applied in the analysis assumes that the particle detected in the Forward Detector is a proton and the $\pi^{-}$ track candidate is selected based on the PID in HADES with a high (> $90 \%$ ) purity. For $20 \%$ to $40 \%$ of events (depending upon which reaction is studied) both daughter particles are emitted into the HADES acceptance and can be easily identified.

However, a small fraction of events with $\Lambda$ decays features a pion registered in the Forward Detector and a proton in HADES or even both particles detected in the Forward Detector. These cases give rise to a small misidentification background (MB), due to the incorrect particle hypothesis in the Forward Detector. There is also a combinatorial background $(\mathrm{CB})$ that arises from combining true protons and pions that do not originate from the decay of a single $\Lambda$. Furthermore, there is physics background (PB) originating from other channels that contain a $\Lambda$ in the final state, but have a much larger production cross-section than the signal channel (see Table 3). Finally, there is also a background originating from combinations of true protons and pions stemming from multi-pion production channels that do not include a $\Lambda$. The rejection strategies adopted to suppress the contributions of these background sources to the final spectra are presented below.

The characteristic delayed decay of the hyperon is used to discriminate the signal from many of these background sources. The typical topology for $\Lambda$ decay following $\Xi^{-}$decay is presented in Fig. 11. This is a more general production scheme than the $\Lambda$ produced in the primary vertex, and illustrates well the observables used for the $\mathrm{CB}$ and $\mathrm{MB}$ suppression.

In the first step a pion and proton are identified and their four-vectors are combined to obtain the four-vector of their mother particle ( $\Lambda$ candidate). The Minimal Track Distance (MTD) between the proton and pion is used to identify a true track pair from the $\Lambda$ decay, for which MTD should ideally be equal to zero. However, the limited track resolution results in a distribution of this observable for track pairs originat-

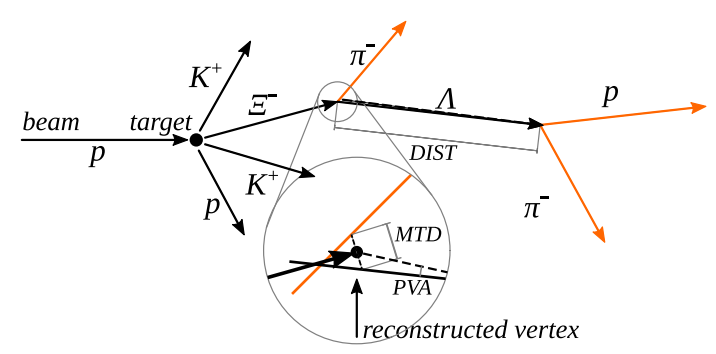

Fig. $11 \Xi^{-}$decay topology and the derived observables. The dots indicate the reconstructed primary vertex and the $\Xi^{-}$decay vertex. The gray lines represent the distance (DIST) between the decay vertices and the minimal track distance (MTD) between the daughter tracks of a given vertex. The Pointing Vector Angle (PVA) is the angle between the reconstructed $\Lambda$ momentum vector and the vector connecting the reconstructed $\Xi^{-}$and $\Lambda$ decay vertices. The orange tracks show particles registered in HADES or the Forward Detector and used in the signal reconstruction

ing from the same vertex. A selection on this variable alone does not adequately discriminate between emission from the primary and the displayed vertices. Nevertheless, it allows to significantly reduce $\mathrm{CB}$ arising from tracks originating from different vertices. Based on the simulations a maximum value for MTD was found to be in the range $15 \mathrm{~mm}$ to $25 \mathrm{~mm}$ and is further adjusted for the specific reaction channel to optimize the signal significance $S / \sqrt{S+B}$, where $S$ is the total yield of signal events and $B$ is the integrated background yield within $\pm 3 \sigma$ of the signal peak. Figure 12(a) shows the distribution of MTD for true $\Lambda$ daughter pairs in the $\Xi^{-}$production channel (solid orange line), $\mathrm{CB}$ and $\mathrm{MB}$ backgrounds from the same final state (dashed yellow line), and random pairs from multi-pion background channels (dotted blue line).

The $\Lambda$ vertex distance (DIST) measures the distance between the $\Lambda$ production vertex and the displaced decay vertex. The primary vertex is located in the $\mathrm{LH}_{2}$ target, which extends from $-55 \mathrm{~mm}$ to $-8 \mathrm{~mm}$ along the beam axis. Requiring a minimum value of DIST reduces the contamination of track pairs resulting from the primary vertex as well as contributions from background channels with $\mathrm{K}_{\mathrm{S}}^{0}$ in the final states, which decays into $\pi^{+} \pi^{-}(c \tau=2.68 \mathrm{~cm})$, where the $\pi^{+}$can be misidentified as a proton. Although this selection discards a fraction of the $\Lambda$ decays close to the primary vertex, the improved $S / B$ ratio is more important, leading to a higher significance for the channel when using this selection. The simulations indicate that the optimal minimum value for DIST should be in the range of $25 \mathrm{~mm}$ to $35 \mathrm{~mm}$ to select $\Lambda$ events. An example of this distribution for the $\Xi^{-}$reaction channel is shown in Fig. 12(b).

The third discriminating observable used in these simulations is the Pointing Vector Angle (PVA), defined as the angle between the reconstructed 3-momentum vector of the $\Lambda$ candidate and the line segment joining the reconstructed produc- 

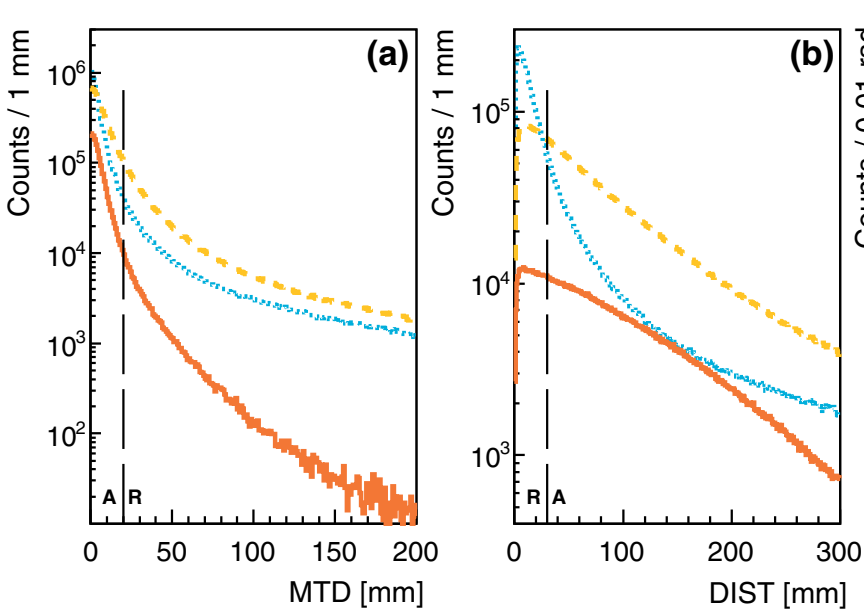

Fig. 12 Examples of the MTD (a), DIST (b) and PVA (c) selections (vertical dashed lines) for the $\mathrm{pp} \rightarrow \Xi^{-} \mathrm{K}^{+} \mathrm{K}^{+} \mathrm{p}$ reaction. The effect of these selections on the $\Lambda$ invariant mass distribution is shown in $(\mathbf{d})$. The solid orange line is for the true $\Lambda$, the dashed yellow is $\mathrm{CB}$ and $\mathrm{MB}$ from the same reaction, and the dotted blue is the distribution for the background stemming from multi-pion production $\mathrm{pp} \rightarrow 2 \mathrm{p} 2 \pi^{+} 2 \pi^{-}$. The vertical line denotes the selections applied in the analysis: the letters $\mathrm{A}$

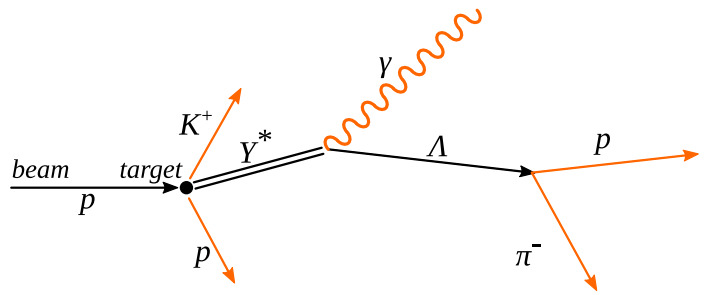

Fig. 13 Topology of hyperon radiative decay. The orange tracks indicate the particles registered in HADES or the Forward Detector and are used in the event reconstruction

tion and decay vertices. The optimal selection on this distribution is in the range 0 to $1 \mathrm{rad}$. It depends on the accuracy of the $\Lambda$ vector reconstruction and the vertex determination, and is correlated with the DIST observable. An example of this selection for the $\Xi^{-}$channel is shown in Fig. 12(c). The second peak near PVA $=\pi$ appears for cases where DIST is small and due to the finite resolution the secondary vertex is reconstructed before the primary vertex $\left(z_{S}<z_{P}\right)$ even though the $\Lambda$ is emitted in the forward direction $\left(p_{z}>0 \mathrm{MeV} / c^{2}\right)$.

Figure 12(d) shows the composite effect of these selections (solid lines) on the reconstructed $\mathrm{p} \pi^{-}$invariant mass distribution for the $\Xi^{-}$signal (red) and the multi-pion background channel (blue). The mass distribution for the $\Xi^{-}$ reaction without these selections (dashed red line) is also scaled to the integral of the distribution after the selections (black dashed). The comparison of the red to the black dashed distributions allow to appreciate the improved $S / B$ ratio.

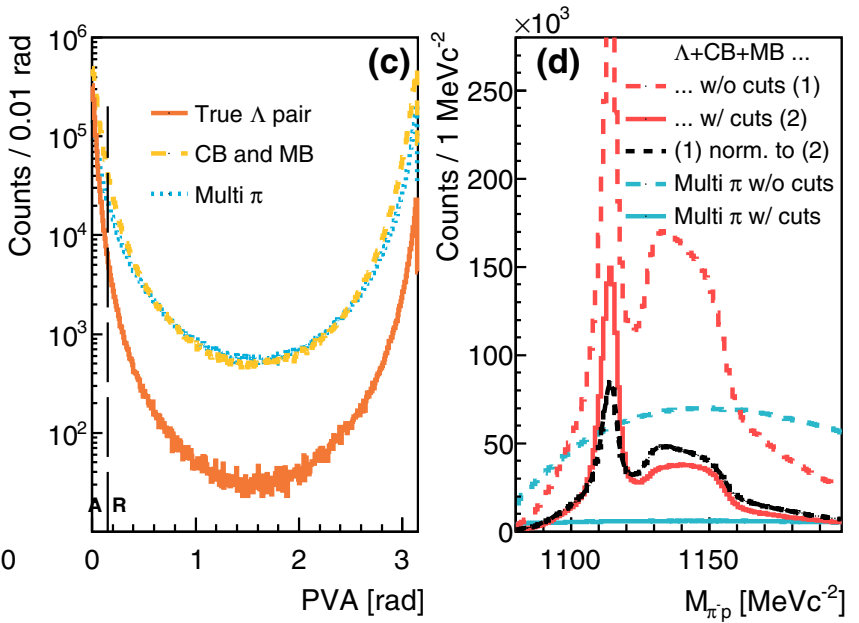

and $\mathrm{R}$ denote the accepted and rejected regions, respectively. In (d), the red line is the reconstructed signal from the $\Xi^{-}$production channel, and the blue line is the distribution for multi-pion production. The dashed line is with no selections applied and the full line is after applying all three selections $(\mathbf{a}-\mathbf{c})$. The black dashed line is the $\Xi^{-}$channel without selections, normalized to the same integral as after the selections. The enhanced $S / B$ ratio is clearly visible

\section{Results}

All simulations used a full cocktail of signal and background channels that are listed in Tables 1 and 3. An arbitrary number of events were simulated, and then weighted according to the corresponding cross-section.

\subsection{Exclusive real photon decay of hyperons}

The topology for $\mathrm{Y} \rightarrow \Lambda \gamma$ is shown in Fig. 13. In contrast to the other analyses presented in this paper, events with real photon decay of hyperons must be reconstructed exclusively in order to suppress the large background of $Y \rightarrow \Lambda \pi^{0}$, where one of the $\pi^{0}$ decay photons is not detected.

The first step in the reconstruction of this channel is the selection of events with the correct final state charged particle candidates, i.e. $\mathrm{p}, \mathrm{p}, \mathrm{K}^{+}$and $\pi^{-}$. In order to obtain very pure lists of particle candidates, deep-learning algorithms with the capability to model complex and non-linear data dependencies are employed here for PID. An Artificial Neural Network (ANN), implemented within the PyTorch framework [63], is used. The network is used as a multi-class classifier to distinguish among five particle species $\mathrm{p}, \pi^{+}, \pi^{-}, \mathrm{K}^{-}$and $\mathrm{K}^{+}$. The input layer consists of seven neurons corresponding to the number of features, which are the three momentum components, the energy loss in the MDC and TOF, as well as the charge and velocity of the particles. The output layer consists of six neurons, five correspond to the particle species and the additional neuron allows the network to classify other particle types as "other particle". The network is trained on the following final states: $\mathrm{pp} \pi^{+} \pi^{-} \pi^{0}, \mathrm{ppK}^{+} \mathrm{K}^{-}$and $\mathrm{pK}^{+} \Lambda \pi^{0}$. 


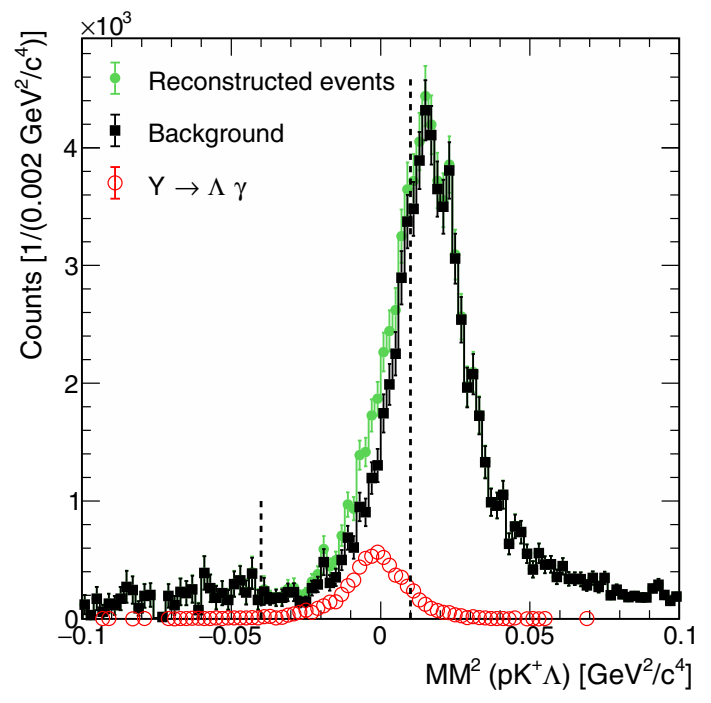

Fig. 14 Distribution of the $\mathrm{pK}^{+} \Lambda$ squared missing mass for reconstructed events in the analysis of the $\Lambda$ radiative decay. The red open circles and black squares show the projected signal and background contributions, respectively. The green circles show the sum. The vertical dashed lines mark the signal region

The best validation accuracy was obtained by sequentially combining three fully connected layers. In addition, a $50 \%$ dropout to each layer was applied to prevent the model from over-fitting. The network converged quickly and has a classification accuracy of $99 \%, 97 \%, 94 \%, 97 \%$ and $93 \%$ for $\mathrm{p}, \mathrm{K}^{+}, \pi^{+}, \pi^{-}$and $\mathrm{K}^{-}$, respectively.

For each event, the point of closest approach of the primary (direct) proton $\mathrm{p}_{\mathrm{p}}$ and the $\mathrm{K}^{+}$candidate was defined as the primary vertex. Since there is more than one proton per event, the MTD between each proton and the $\mathrm{K}^{+}$tracks is calculated. The proton and corresponding primary vertex with the smallest MTD is selected. Furthermore the primary vertex is required to be located within the target volume $(-60 \mathrm{~mm}<z<-5 \mathrm{~mm}$, and $r<6 \mathrm{~mm})$.

The intermediate $\Lambda$ has been reconstructed by demanding that its decay products (i.e. $\mathrm{p}$ and $\pi^{-}$) originate from a delayed vertex. All combinations of $\mathrm{p}$ and $\pi^{-}$candidates are used to form $\Lambda$ candidates. For each $\Lambda$ candidate the decay vertex is defined as the Point of Closest Approach between the two tracks. If both tracks are in HADES, then the MTD between the $\mathrm{p}$ and $\pi^{-}$tracks is required to be smaller than $15 \mathrm{~mm}$. If a track is measured in the Forward Detector it is assumed to be a proton and the MTD is required to be less than $20 \mathrm{~mm}$. The boost of the $\Lambda$ into the laboratory reference frame ensures that the $\Lambda$ and its decay proton are almost collinear, while the $\pi^{-}$should have a larger deviation from the direction of the $\Lambda$ momentum vector. Thus, the Distance of Closest Approach between the p track and the primary vertex is required to be smaller than for the $\pi^{-}$track and the primary vertex. Furthermore, the PVA is required to be smaller than $0.5 \mathrm{rad}$.

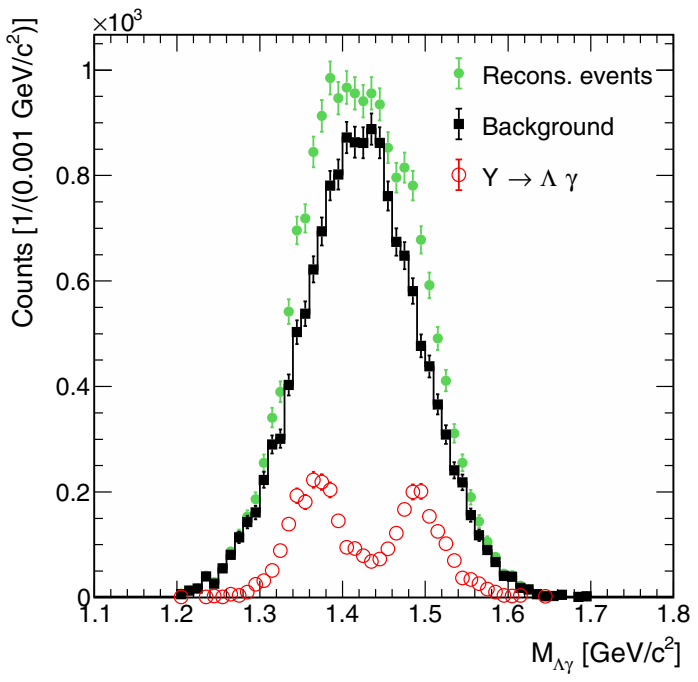

Fig. 15 Projected $\Lambda$ invariant mass distribution of the reconstructed events in the signal region (the green circles). The black squares are background channels and the red open circles is the $\mathrm{Y} \rightarrow \Lambda \gamma$ signal after background subtraction. The contributions are scaled according to the expected integrated luminosity corresponding to 28 days of measurement with the $\mathrm{LH}_{2}$ target

Photon candidates are identified as an energy cluster in the ECAL above a certain threshold. The contribution from charged particles is suppressed by requiring the cluster to be spatially uncorrelated with the RPC hits. The 4-vector of the candidate photon is calculated from the cluster energy and the cluster position [64]. Photon candidates are required to have $0.96<\beta<1.04$, and an energy deposition above $0.35 \mathrm{GeV}$.

Since several background channels mimic the signal, two kinematic variables have been used for further background suppression: The $\mathrm{p}_{\mathrm{p}} \mathrm{K}^{+}$squared missing mass is required to be in the range $1.6<M M^{2}\left(\mathrm{p}_{\mathrm{p}} \mathrm{K}^{+}\right)<2.6 \mathrm{GeV}^{2} / c^{4}$. The squared $\mathrm{p}_{\mathrm{p}} \mathrm{K}^{+} \Lambda$ missing mass $\left(M M^{2}\left(\mathrm{p}_{\mathrm{p}} \mathrm{K}^{+} \Lambda\right)\right)$ should peak at zero for signal events, since only one photon is missing. Thus it is required to be in the range $-0.04<$ $M M^{2}\left(\mathrm{p}_{\mathrm{p}} \mathrm{K}^{+} \Lambda\right)<0.01 \mathrm{GeV}^{2} / c^{4}$. The signal range is asymmetric about zero in order to reduce contamination from the $\mathrm{pK}^{+} \Lambda \pi^{0}$ background, which is peaked at the mass squared of the $\pi^{0}$. The results are shown in Fig. 14, where the red open circles indicate the signal, the black squares the background, and the sum of both is shown as the green circles. The vertical dashed lines indicate the region of the squared missing mass selected for the signal.

Finally, the excited hyperon is reconstructed in the $\Lambda \gamma$ invariant mass distribution and presented in Fig. 15. The background is mostly from the $\mathrm{pK}^{+} \Lambda \pi^{0}$ channel (reaction 11 in Table 3). The slight shift in the peak position of the $\Sigma(1385)^{0}$ and $\Lambda(1520)$ results from the response function of the ECAL. The estimated overall acceptance times reconstruction efficiency for $\Sigma(1385)^{0}, \Lambda(1405)$ and $\Lambda(1520)$ is 


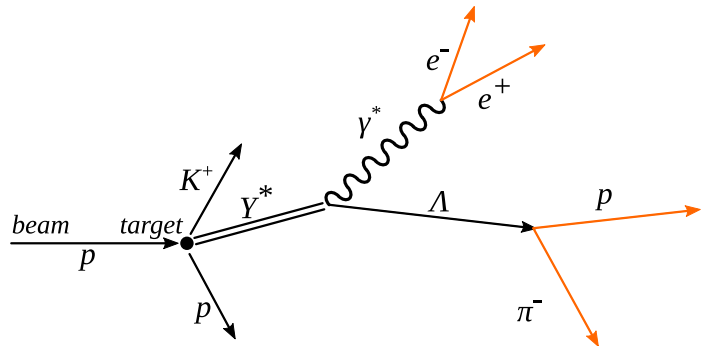

Fig. 16 Topology of hyperon Dalitz-decay. The orange tracks indicate the particles registered in HADES and used in the reconstruction

$0.030 \%, 0.030 \%$ and $0.026 \%$, respectively. The corresponding significance to reconstruct each of these states is of 16 , 0.3 and 15.

\subsection{Dalitz-decay of excited hyperons}

The Dalitz-decay of hyperons (Fig. 16) was reconstructed in the reactions where the primary hyperon resonance decays into a $\Lambda$ and a virtual photon $\gamma^{*}$, which then decays into an $\mathrm{e}^{+} \mathrm{e}^{-}$dilepton pair. The inclusive reconstruction of $\Lambda$ candidates proceeded similar to the $\Xi^{-}$channel. The main difference is that the decays of the hyperon resonances occurs at the primary vertex. The subsequent $\Lambda$ decay appears at a displaced vertex. Therefore, the $\mathrm{e}^{+} \mathrm{e}^{-}$pair originates at the primary vertex located in the target. The $\mathrm{z}$-coordinate of the secondary vertex is required to be $>0 \mathrm{~mm}$, for the given target position extending from -55 to $-8 \mathrm{~mm}$. Furthermore, a MTD $<20 \mathrm{~mm}$ was demanded for $\Lambda$ candidates to reduce the background from uncorrelated pion-proton pairs. The minimal opening angle for the dilepton pair is $4^{\circ}$ to reduce conversion background, which is mostly emitted at lower opening angles.

Figure 17(a) shows the reconstructed $\mathrm{e}^{+} \mathrm{e}^{-}$invariant mass spectrum. The combinatorial background (CB) originating from uncorrelated $\mathrm{e}^{+} \mathrm{e}^{-}$pairs is shown by the red dots. The magenta dots represent the sum of all reconstructed Dalitz $\mathrm{e}^{+} \mathrm{e}^{-}$signal. The blue-green histogram represent $\mathrm{e}^{+} \mathrm{e}^{-}$pairs that originate from $\pi^{0}$ decays. Dalitz-decays of the $\Delta$ are denoted by the blue histogram. The yellow and green histograms show the spectra originating from hyperon $(\Lambda(1520)$ and $\Sigma(1385)$, respectively) Dalitz-decays. The figure clearly shows that the region of invariant mass below $140 \mathrm{MeV} / c^{2}$ is dominated by the $\pi^{0}$ Dalitz decay, which can not be fully suppressed by the conditions on the $\Lambda$ reconstruction. Above the $\pi^{0}$ range, the main background comes from the $\Delta$ Dalitz decay.

Figure 17(b) shows the $\Lambda \mathrm{e}^{+} \mathrm{e}^{-}$invariant mass distribution, where the dilepton mass is required to be above the $\pi^{0}$ mass (i.e. $M_{\mathrm{e}}{ }^{+} \mathrm{e}^{-}>140 \mathrm{MeV} / c^{2}$ ). Clear peaks from the $\Lambda(1520)$ and $\Sigma(1385)^{0}$ are visible above a broad background from the $\Delta$. The low branching ratio for the $\Lambda(1405)$ results in too little yield to be measured here. The product of the acceptance times reconstruction efficiency is estimated to be about $0.48 \%$ and $0.58 \%$ for the $\Sigma(1385)^{0}$ and $\Lambda(1520)$, respectively.

These simulations were performed under the assumption that the decaying particles are point-like. As discussed in the introduction, it is expected that the form factors will enhance the decay rate in the high mass region and consequently increase the count rates with respect to these simulations. The expected count rates are summarized in Table 4.

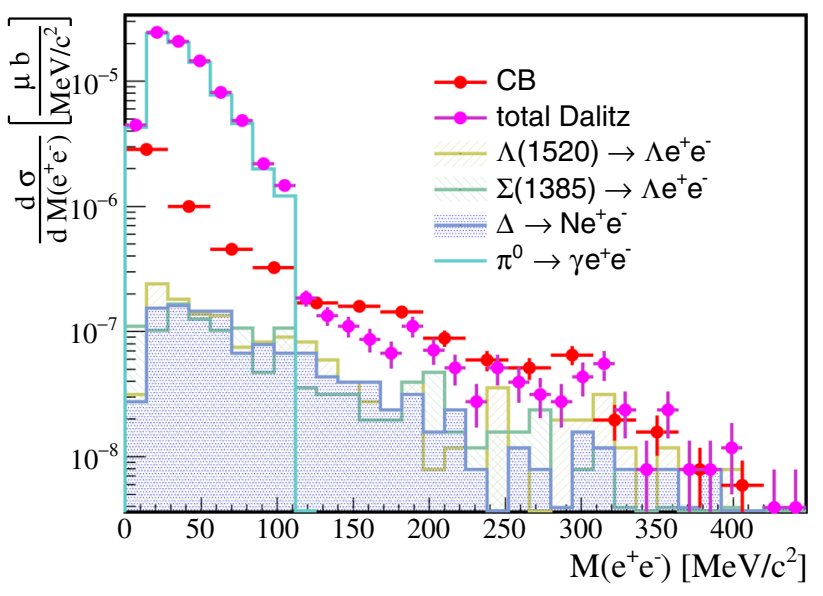

(a)

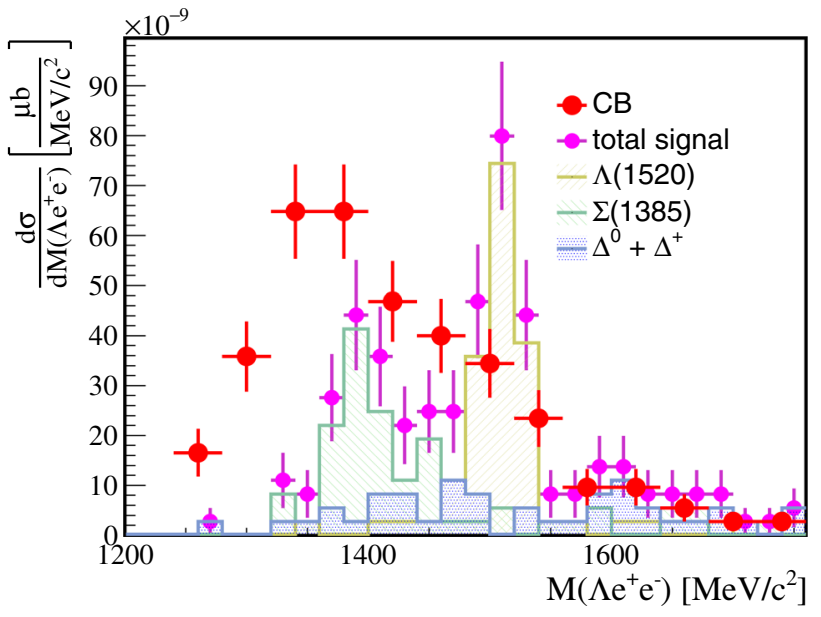

(b)

Fig. 17 Dilepton invariant mass spectrum from signal $\gamma^{*}$ and background $\pi^{0}$ sources (a) and reconstructed $\Lambda(1520), \Sigma(1385)$ and $\Lambda(1405)$ peaks (b), see text for details. The statistical uncertainty corresponds to a four week measurement at a luminosity of $1.5 \times 10^{31} /\left(\mathrm{cm}^{2} \mathrm{~s}\right)$ 


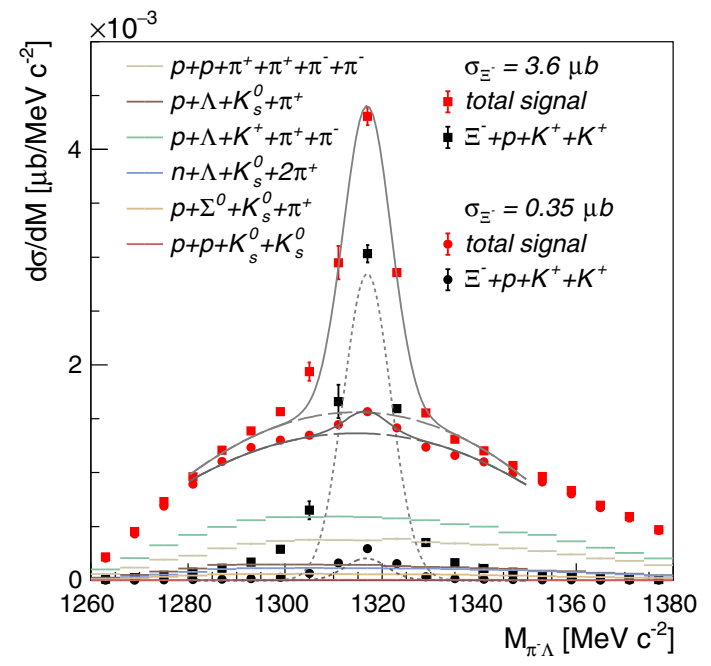

Fig. 18 Reconstructed $\Lambda \pi^{-}$invariant mass spectrum. The red points indicate the distributions for a $\Xi^{-}$signal cross-section of $3.6 \mu \mathrm{m}$ and $0.35 \mu \mathrm{m}$. The black points indicate the corresponding distributions after the combinatorial background (CB) has been subtracted

\subsection{Inclusive $\Xi^{-}$production}

The $\Xi^{-}$decay was reconstructed by the following chain of weak decays: in the first step the $\Xi^{-}$decays into a $\Lambda \pi^{-}$ pair and in the second step the $\Lambda$ decays into a $\mathrm{p} \pi^{-}$pair. The $\Lambda$ was reconstructed as described above, considering pion tracks from HADES and proton tracks identified in either HADES or the Forward Detector. The $\Xi^{-}$was reconstructed combining $\Lambda$ candidates and pions from HADES. In order to effectively reduce the combinatorial background from the signal and the misidentification background, the following set of topological selections has been applied (Fig. 11): (a) $\mathrm{MTD}_{\Lambda}$ for the proton and pion track candidates from $\Lambda$ decays is required to be $<25 \mathrm{~mm},(b)$ the $\mathrm{z}$-coordinate for the $\Lambda$ decay vertex is required to be in the range $-20 \mathrm{~mm}<z_{\Lambda}<300 \mathrm{~mm}$, (c) MTD $_{\Xi}^{-}$between the $\Lambda$ and the pion track candidates from $\Xi^{-}$decay is required to be $<20 \mathrm{~mm},(d)$ the PVA of $\Lambda$ track to the line between $\Xi^{-}$and $\Lambda$ decay vertices is lower than $0.15 \mathrm{rad}$.

An additional selection on the squared missing mass of the $\Xi^{-}$candidate relative to the beam+target system $M M^{2}>2050^{2} \mathrm{MeV}^{2} / c^{4}$ has been applied to suppress background channels. The specific selection values have been chosen to optimize the significance. To reduce misidentifying pions and kaons as protons in the Forward Detector, only those tracks with $t_{\text {tof }}>27 \mathrm{~ns}$ (Fig. 10) are accepted. The overall acceptance times reconstruction efficiency for the $\Xi^{-}$is determined to be about $1.68 \%$.

Two values of the $\Xi^{-}$production cross-sections $(3.6 \mu \mathrm{b}$ and $0.35 \mu \mathrm{b}$ ) have been used for these simulations. The resulting $\Lambda \pi^{-}$invariant mass distributions are shown in Fig. 18, together with various background channels (denoted
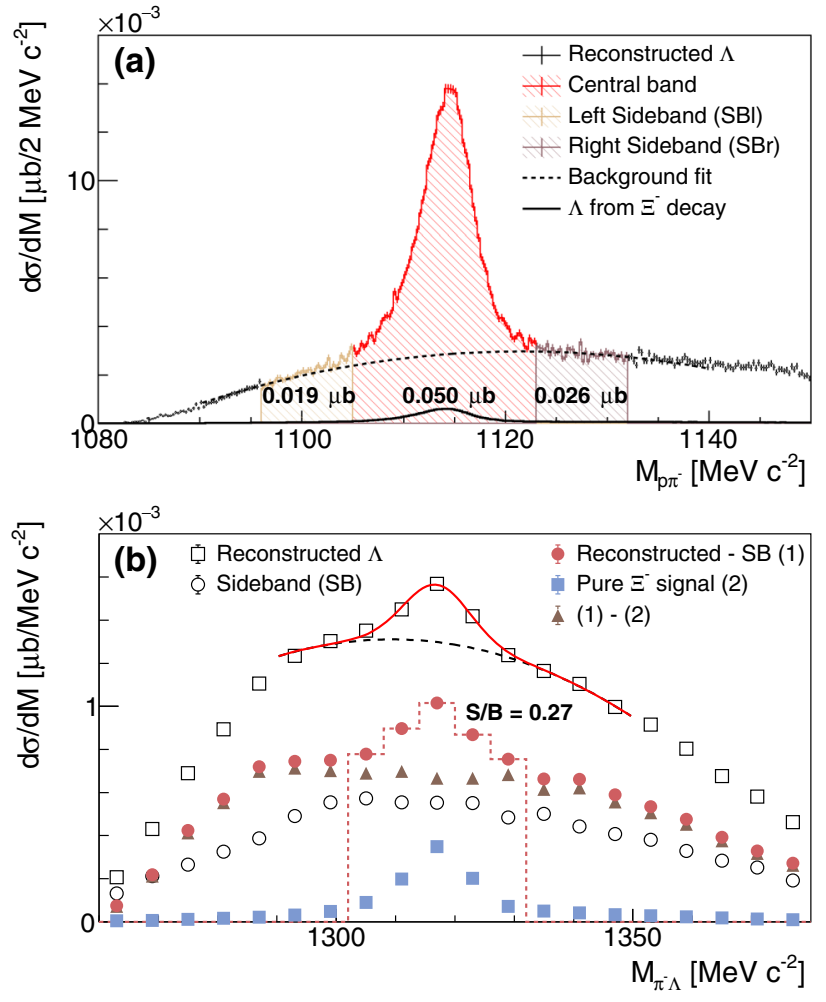

Fig. 19 Sideband analysis for the $\Xi^{-}$signal. (a) Sideband regions for the daughter $\Lambda$ invariant mass spectrum. The dashed line shows a fit to estimate the background under the signal peak. The numbers show the integrated cross-section values of the background for each background region. (b) $\Lambda \pi^{-}$invariant mass spectra. The open square symbols show the distribution for all signal candidates. The open circles represent the spectrum for the $\Lambda$ sideband region, and the solid circles are the full results after subtracting the sideband region. The solid square symbols show the $\Xi^{-}$signal for reference. The brown triangles mark the estimated background line shape

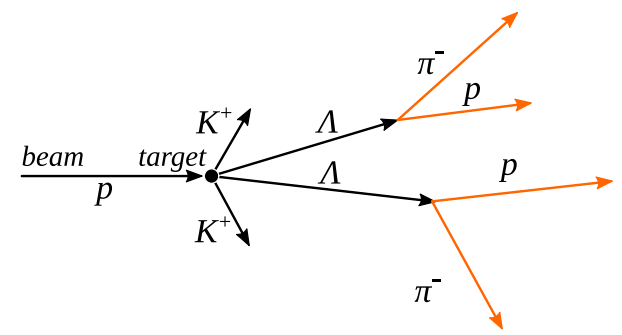

Fig. $20 \Lambda \Lambda$ decay topology. The orange tracks indicate the particles registered in HADES and used in the reconstruction

by different colored lines). For the case with the higher cross-section (red squares) the $\Xi^{-}$peak is clearly visible on the top of the background. For the other case (red circles) the $\Xi^{-}$peak is less pronounced. For the reference, the true $\Xi^{-}$signal is shown for both cases by the black symbols. The full spectrum for each case was fit with the sum of a fifth order polynomial for the background and a Gaussian for the signal. These fits are drawn as solid gray lines and the back- 
ground contribution is drawn as long-dashed lines. The mass of the $\Xi^{-}$peak is found to be $1317.77(62) \mathrm{MeV} / c^{2}$. It differs slightly from the PDG value of $1321.71(7) \mathrm{MeV} / c^{2}$. Also the peak of the pure $\Xi^{-}$distribution is asymmetric. This asymmetry in the line shape and the shift of the reconstructed peak position result from Forward Detector tracks which require further corrections, such as for energy loss in the detector material and the different velocities of the hyperon and its daughter proton. The statistics presented in this figure corresponds to 2.5 days of data taking with the $\mathrm{LH}_{2}$ target.

In order to reduce the background under the $\Xi^{-}$peak due to uncorrelated $\pi^{-} p$ pairs, a sideband analysis of the $\pi^{-} p$ invariant mass spectrum has been used (see Fig. 19(a)). This background is related to contributions from reactions without $\Lambda$ production. A region on the left $1096 \mathrm{MeV} / c^{2}$ to $1105 \mathrm{MeV} / c^{2}$ and one on the right $1123 \mathrm{MeV} / c^{2}$ to $1132 \mathrm{MeV} / c^{2}$ were used to estimate the background. The selected sidebands have the same width as the $\Lambda$ peak region and their integrated yield is slightly smaller than the signal region due to the non-linear shape of the background. The background yield under the peak was estimated from a fit, presented by the dashed black line. Since only a small fraction of the $\Lambda \mathrm{s}$ in the peak originate from the $\Xi^{-}$signal (solid black line), only a fraction of the background under the $\Xi^{-}$ signal can be removed by this sideband analysis.

For each sideband the $\mathrm{p} \pi^{-} \pi^{-}$invariant mass distribution was calculated just as for the $\Lambda$ peak region. The sum of the resulting distributions (open circles in Fig 19b) was scaled to the yield of the background under the $\Lambda$ peak (region below the dashed line in the central region in Fig. 19a) and subtracted from the $\mathrm{p} \pi^{-} \pi^{-}$invariant mass distribution for the $\Lambda$ peak region (open squares). The resulting $\Lambda \pi^{-}$invariant mass distribution is presented by the solid red circles. For

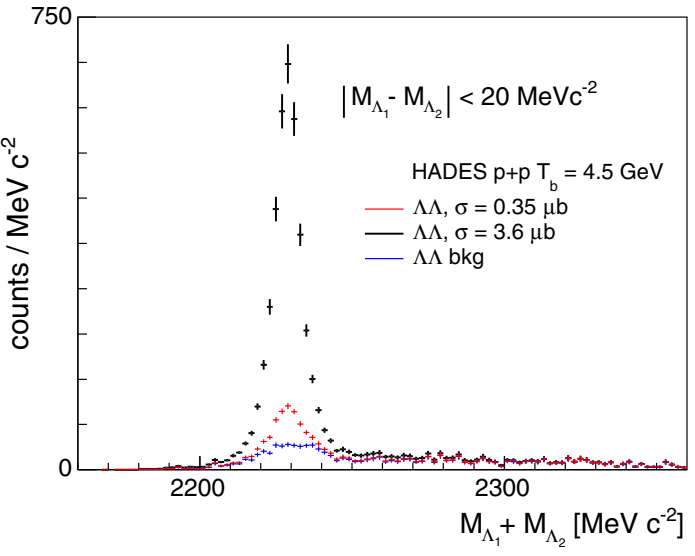

Fig. 22 Sum of the invariant masses for both $\Lambda$ candidates in $\Lambda \Lambda$ decay for the two $\Lambda \mathrm{s}$ mass difference less than $20 \mathrm{MeV} / c^{2}$

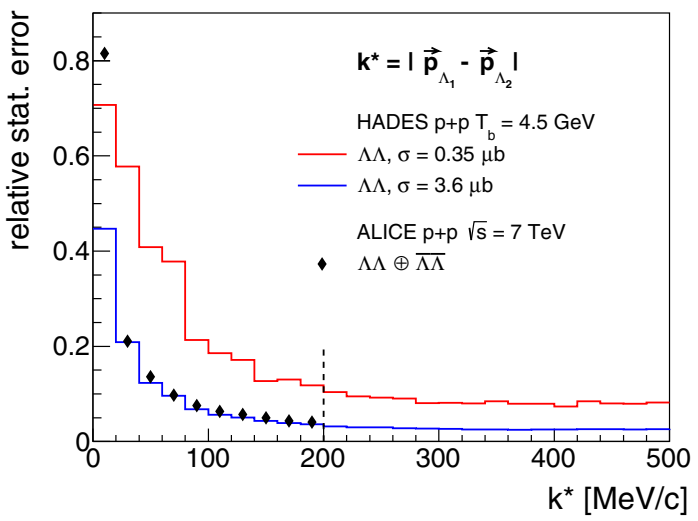

Fig. 23 Relative error of the yield for $\Lambda \Lambda$ pairs as a function of the relative momentum
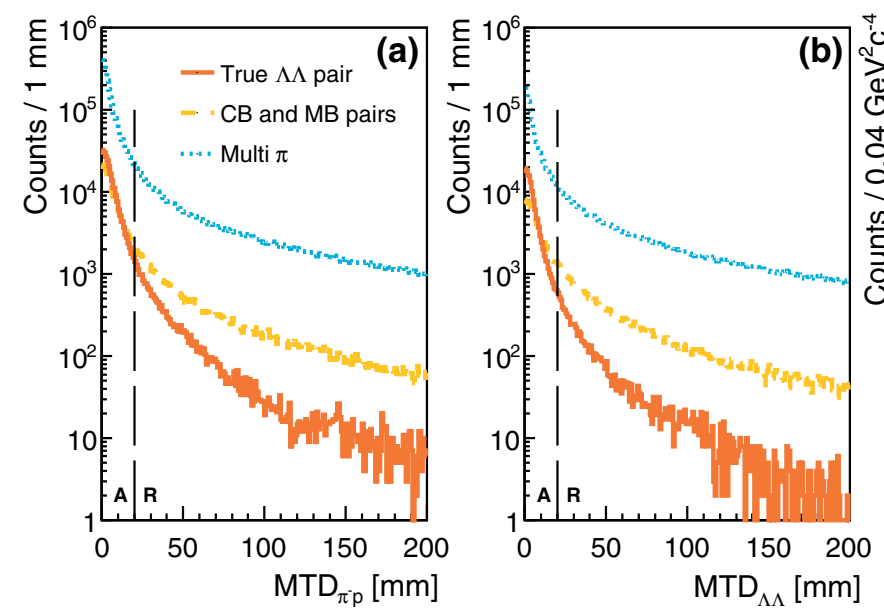

Fig. 21 The $\mathrm{MTD}_{\pi^{-} \mathrm{p}}$ distribution for $\Lambda$ daughters in $\Lambda \Lambda$ events (a), the $\mathrm{MTD}_{\Lambda \Lambda}$ distribution for the two $\Lambda$ candidates (b), the squared missing mass $\mathrm{MM}^{2}$ value (c) and the PVA values for both $\Lambda$ candi-
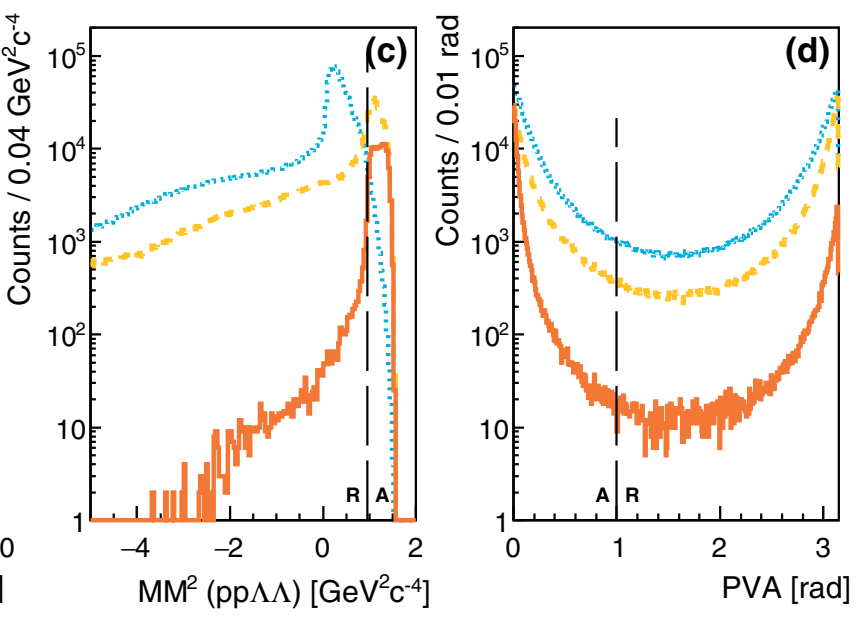

dates (d). The vertical lines mark the accepted and rejected regions, denoted by the letters A and R, respectively 
Table 4 Expected count rates for luminosity $\mathcal{L}=1.5 \times 10^{31} /\left(\mathrm{cm}^{2} \mathrm{~s}\right)$ available using a liquid hydrogen $\left(\mathrm{LH}_{2}\right)$ target and a polyethylene $\left(\mathrm{CH}_{4}\right.$ target of the same dimensions. The branching ratios include a factor of 0.64 for each $\Lambda \rightarrow \mathrm{p} \pi^{-}$decay included

\begin{tabular}{llllll}
\hline decay process & $\sigma[\mu \mathrm{b}]$ & BR & $\epsilon \cdot$ acc $[\%]$ & counts/day $\left(\mathrm{LH}_{2}\right)$ & counts/day $(\mathrm{CH})$ \\
\hline$\Sigma(1385)^{0} \rightarrow \Lambda \mathrm{e}^{+} \mathrm{e}^{-}$ & 56 & $8.94 \times 10^{-5}$ & 0.48 & 15 & 105 \\
$\Lambda(1520) \rightarrow \Lambda \mathrm{e}^{+} \mathrm{e}^{-}$ & 69 & $6.93 \times 10^{-5}$ & 0.58 & 18 & 126 \\
$\Lambda(1520) \rightarrow \Lambda \pi^{-} \pi^{+}$ & 69 & $4.22 \times 10^{-2}$ & 1.4 & $2.64 \times 10^{4}$ & $1.85 \times 10^{5}$ \\
$\Sigma(1385)^{0} \rightarrow \Lambda \gamma$ & 56 & $9.07 \times 10^{-3}$ & 0.030 & 99 & 692 \\
$\Lambda(1520) \rightarrow \Lambda \gamma$ & 69 & $7.03 \times 10^{-3}$ & 0.026 & 82 & 574 \\
$\Xi^{-} \rightarrow \mathrm{p} \pi^{-} \pi^{-}$ & 3.6 & 0.64 & 1.68 & $2.43 \times 10^{4}$ & $1.70 \times 10^{5}$ \\
$\Xi^{-} \rightarrow \mathrm{p} \pi^{-} \pi^{-}$ & 0.35 & 0.64 & 1.68 & $2.43 \times 10^{3}$ & $1.70 \times 10^{4}$ \\
$\mathrm{pp} \rightarrow \Lambda \Lambda \mathrm{K}^{+} \mathrm{K}^{+}$ & 3.6 & $0.64^{2}$ & 0.34 & $3.15 \times 10^{3}$ & $2.20 \times 10^{4}$ \\
$\mathrm{pp} \rightarrow \Lambda \Lambda \mathrm{K}^{+} \mathrm{K}^{+}$ & 0.35 & $0.64^{2}$ & 0.34 & $3.15 \times 10^{2}$ & $2.20 \times 10^{3}$ \\
\hline
\end{tabular}

comparison the true $\Xi^{-}$signal marked by the blue squares is also drawn.

The region marked by the dashed red line was then used to extract the $S / B$ ratio and the significance. The total yield in this mass region minus the pure $\Xi^{-} \operatorname{signal}(S)$ (blue squares) defines the background ( $B$ ) (solid triangles). The $S / B$ ratio is 0.27 and the estimated significance in this invariant mass region for one day of data taking is estimated to be 28 , allowing for the $\Xi^{-}$to be reconstructed and the production crosssection to be determined.

\section{$5.4 \Lambda \Lambda$ production}

The topology of double $\Lambda$ production is shown in Fig. 20. Both $\Lambda \mathrm{s}$ are emitted directly from the primary reaction vertex. The reconstruction procedure does not distinguish the $\Lambda$ particles, but double counting is avoided, as described below. Reconstruction of the $\Lambda$ decays follows the standard $\Lambda$ procedure described in Sect. 4.4 with $\mathrm{MTD}_{\pi^{-}}$p for both $\Lambda$ s set to $20 \mathrm{~mm}$. To reduce $\mathrm{CB}$ and $\mathrm{MB}$, three additional selections on the two $\Lambda$ systems were applied: (a) missing mass of ( pbeam $_{\text {berget }}, \Lambda, \Lambda$ ) $>980 \mathrm{MeV} / c^{2}, a$ ) minimal track distance between both $\Lambda \mathrm{s} \mathrm{MTD}_{\Lambda \Lambda}<20 \mathrm{~mm}$ and $a$ ) the PVA was required to be less than $1.0 \mathrm{rad}$. The effect of these selections is shown in Fig. 21 for the $\mathrm{pp} \rightarrow \Lambda \Lambda \mathrm{X}$ as well as the $\mathrm{CB}, \mathrm{MB}$ and multipion backgrounds. The estimated overall acceptance times reconstruction efficiency for this channel is $0.34 \%$.

Events with $\Lambda$ pairs are selected by requiring at least two distinct $\Lambda$ candidates in the event, and that the difference between their invariant masses is smaller than $20 \mathrm{MeV} / c^{2}$. The distribution of the sum of the candidates masses is shown in Fig. 22, where both cross-section estimates investigated have been considered (black and red points). The common background for both cases is shown in blue. This background originates from events of reactions 17-20, 22 (Table 3) with one $\Lambda$ and the second candidate is contained in the mass window by chance.

Events containing two $\Lambda$ s open the possibility to study $\Lambda \Lambda$ correlations. Based on the overall acceptance times reconstruction efficiency determined above $(0.34 \%)$, we expect an integrated yield of 220-2200 reconstructed events per day, depending on the assumed production cross-section.

The corresponding statistical precision for the relative momentum spectrum $\left(k^{*}\right)$ is shown in Fig. 23 for both crosssection estimates and is compared to the published values from ALICE. Information on the $\Lambda \Lambda$ interaction can be primarily gained in the low $k^{*}$ region. We expect 312 and 3120 events with $k^{*}<0.2 \mathrm{GeV} / c$ in a 4 week experiment for the two scenarios. The yield and statistical precision are expected to be similar to the results from ALICE for the upper bound cross-section. More importantly, even for the lower bound cross-section, the expected precision is better in HADES in the region with $k^{*}<20 \mathrm{GeV} / c$, which is most sensitive to the interaction parameters. Systematic uncertainty due to the large freeze-out volume and feed-down effects are important limitations for the existing results and those effects are expected to be significantly smaller for HADES with $\mathrm{p}+\mathrm{p}$ at $E_{\text {kin }}=4.5 \mathrm{GeV}$.

\subsection{Count rate estimates}

The count rates for these studies have been calculated based on the following parameters: $(a)$ the reconstruction efficiency times acceptance for the various channels, as obtained from the simulation studies described above, $(b)$ a beam duty cycle of $50 \%$, and (c) two options for the maximum luminosity as described in Section 2

Table 4 summarizes all the channels considered. The production cross-sectionsfor the proton-proton reactions and the branching ratios are given in columns 2 and 3 . The product of the acceptance and reconstruction efficiencies is given in 
column 4. Columns 5 and 6 give the expected count rates for the $\mathrm{LH}_{2}$ and the PE targets, respectively.

\section{Summary}

Dalitz decays of hyperons is an experimentally unexplored process, which can be studied for the first time by using the excellent capabilities of HADES to measure rare dilepton decays. Such measurements will provide complementary information on hyperon structure and the role of strange quarks in baryons. The latter will be investigated by comparing these results to those transitions recently measured for non-strange baryons of same spin and parity (i.e. $\Sigma(1385)^{0}$ vs $\Delta(1232)$ and $\Lambda(1520)$ vs $\left.N^{*}(1520)\right)$.

Production cross sections of the higher mass hyperon resonances investigated here are unknown in the energy range of future FAIR experiments. Hence, the proposed measurements will provide an essential input for the understanding of the production mechanism in proton-proton interaction in this energy regime and will also establish an important reference for $\mathrm{p}+\mathrm{A}$ and $\mathrm{A}+\mathrm{A}$ collisions. In particular, the production of double strangeness offers a unique opportunity to study strangeness production close to the threshold, where only few production channels are expected to contribute. In this regime, characterized by a small relative momentum between the produced hadrons, final state interactions are expected to play a significant role. Therefore, hyperonhyperon and nucleon-hyperon correlation studies are very promising tools to measure their interaction potential.

The feasibility studies presented here show that all the benchmark channels with hyperon Dalitz-decays, except $\Lambda(1405)$, can be measured in HADES including the new Forward Detector, which has been described in detail in this paper. The biggest uncertainty in these results comes from scarce cross-sectiondata for various signal and background channels. Thus, a systematic error of $40 \%$ is assigned to the count rates for most channels. However, the crosssectionestimates for the double strange baryons $\Xi^{-}$and $\Lambda \Lambda$ are sensitive to unknown factors and have much larger uncertainty, up to an order of magnitude. The predicted count rates for the electromagnetic decays of $\Sigma(1385)^{0}$ and $\Lambda$ (1520) favor the use of the polyethylene target combined with a high luminosity operating mode that will become possible with an upgraded HADES DAQ. For all benchmark channels, four weeks of measurement time is considered sufficient to measure the corresponding cross-sections.

Currently, the HADES collaboration discusses a possible extension of the tracking region to angles $7^{\circ}$ to $18^{\circ}$ allowing to cover blind spots of the spectrometer. Furthermore, a fast trigger hodoscope in front of the inner MDC is considered for a better on-line event selection. Such improvements will result in a further significant extension of the acceptance, as seen in Fig. 8, and better trigger possibilities.

Acknowledgements We gratefully acknowledge financial support by the Polish National Science Centre, Grant 2016/23/P/ST2/04066 POLONEZ funded from the European Union's Horizon 2020 research and innovation program under the Marie Skodowska-Curie Grant agreement No. 665778. The collaboration gratefully acknowledges the support by SIP JUC Cracow, Cracow (Poland), National Science Center, 2017/25/N/ST2/ 00580, 2017/26/M/ST2/00600; Forschungszentrum Jülich, Jülich (Germany); TU Darmstadt, Darmstadt (Germany), VHNG-823, BMBF: 05P18RDFC1, DFG GRK 2128, DFG CRC-TR 211; Goethe-University, Frankfurt (Germany) and TU Darmstadt, Darmstadt (Germany), ExtreMe Matter Institute EMMI at GSI Darmstadt; TU München, Garching (Germany), MLL München, DFG EClust 153, GSI TMLRG1316F, BMBF 05P15WOFCA, SFB 1258, DFG FAB898/ 2-2; NRNU MEPhI in the framework of the Russian Academic Excellence Project (contract No. 02.a03.21.0005, 27.08.2013), RFBR funding within the research project no. 18-02-40086, Ministry of Science and Higher Education of the Russian Federation, Project "Fundamental properties of elementary particles and cosmology" No 0723-2020-0041, JLU Giessen, Giessen (Germany), BMBF:05P12RGGHM; IPN Orsay, Orsay Cedex (France), CNRS/IN2P3; NPI CAS, Rez, Rez (Czech Republic), MSMT LM2018112, OP VVV CZ.02.1.01/0.0/0.0/16013/00 01677, LTT17003.

Funding Open Access funding enabled and organized by Projekt DEAL.

Data Availability Statement This manuscript has no associated data or the data will not be deposited. [Authors' comment: This is a simulation study. Therefore there are no data. However, on request the authors can make the simulated data points available.]

\section{References}

1. G. Agakishiev et al., Eur. Phys. J. A 41, 243 (2009)

2. J. Adamczewski-Musch et al., Phys. Rev. C 95, 065205 (2017)

3. B. Ramstein et al., EPJ Web Conf. 199, 01008 (2019)

4. J. Adamczewski-Musch et al., Phys. Rev. C 102, 024001 (2020)

5. G. Agakishiev et al., Eur. Phys. J. A 51, 137 (2015)

6. R. Münzer et al., Phys. Lett. B 785, 574 (2018)

7. G. Agakishiev et al., Phys. Lett. B 750, 184 (2015)

8. J. Adamczewski-Musch, O. Arnold, C. Behnke et al., Nat. Phys. 15, 1040 (2018)

9. P. Salabura et al., EPJ Web Conf. 241, 01013 (2020)

10. G. Ramalho, M.T. Pena, Phys. Rev. D 85, 113014 (2012)

11. G. Ramalho, Phys. Rev. D 100, 114014 (2019)

12. I.G. Aznauryan, V.D. Burkert, Prog. Part. Nucl. Phys. 67, 1 (2012)

13. E. Kaxiras, E.J. Moniz, M. Soyeur, Phys. Rev. D 32, 695 (1985)

14. M. Tanabashi et al., Phys. Rev. D 98, 030001 (2018)

15. S. Taylor et al., Phys. Rev. C 71, 054609 (2005)

16. D. Keller et al., Phys. Rev. D 85, 052004 (2012)

17. G. L. Landsberg, V. V. Molchanov, Radiative decays of hyperons, tech. rep. IHEP-97-42 (Protvino Inst. High Energy Phys., Protvino, July 1997)

18. S. Dobbs et al., Phys. Lett. B 739, 90 (2014)

19. C. Granados, S. Leupold, E. Perotti, Eur. Phys. J. A 53, 117 (2017)

20. G. Ramalho, K. Tsushima, Phys. Rev. D 84, 054014 (2011)

21. G. Ramalho, PRD 102, 054016 (2020)

22. G. Ramalho et al., Phys. Rev. D 93, 033004 (2016)

23. R.A. Williams, C.-R. Ji, S.R. Cotanch, Phys. Rev. C 48, 1318 (1993) 
24. P. Alba, et al., Workshop on Excited Hyperons in QCD Thermodynamics at Freeze-Out (YSTAR016) (2017). arXiv:1701.07346 [hep-ph]

25. B. Friman, et al., eds., The CBM physics book: Compressed baryonic matter in laboratory experiments, Vol. 814 (2011)

26. G. Agakishiev et al., Phys. Rev. Lett. 103, 132301 (2009)

27. G. Agakishiev et al., Phys. Rev. Lett. 114, 212301 (2015)

28. J. Steinheimer, M. Bleicher, J. Phys. G 43, 015104 (2016)

29. M. Zetenyi, G. Wolf, Phys. Lett. B 785, 226 (2018)

30. G. Agakishiev et al., Phys. Rev. C 85, 035203 (2012)

31. S. Acharya et al., Phys. Rev. C 99, 024001 (2019)

32. N.K. Glendenning, APJ 293, 470 (1985)

33. K. Peters, et al., Nucl. Phys. News 27, 24 (2017)

34. P. Salabura, J. Stroth, Dilepton Radiation from Strongly Interacting Systems (2020). arXiv:2005.14589 [nucl-ex]

35. U. Mosel, V. Metag, Nucl. Phys. News 3, 25 (1993)

36. FAIR - The Universe in the Lab. https://fair-center.eu

37. W. Erni et al., Eur. Phys. J. A 49, 25 (2013)

38. J. Smyrski et al., J. Instrum. 13, P06009 (2018)

39. B. Ramstein, EPJ Web Conf. 241, 01012 (2020)

40. F. Scozzi, Studying excited states of the nucleon with the HADES detector at gsi. PhD thesis (Technische Universität Darmstadt, Darmstadt, pp. 109-112 (2018)

41. P. Wintz, Hyperfine Interact. 229, edited by T. Johansson, P. Froehlich, and S. Jonsell, 147 (2014)

42. J. Smyrski et al., J. Instrum. 12, C06032 (2017)

43. D. Przyborowski et al., J. Instrum. 11, P08009 (2016)

44. M. Traxler et al., J. Instrum. 6, C12004 (2011)

45. S. Jowzaee et al., Nucl. Instrum. Methods. A 718, 573 (2013)

46. P. Strzempek, Development and evaluation of a signal analysis and a readout system of straw tube detectors for the PANDA spectrometer. PhD thesis (Jagiellonian University, Krakow, Poland, 2017)
47. C. Finck, P. Fonte, A. Gobbi, Nucl. Instrum. Methods. A 508, 63 (2003)

48. D. Belver et al., Nucl. Instrum. Methods. A 602, 687 (2009)

49. D. Belver et al., IEEE Trans. Nucl. Sci. 57, 2848 (2010)

50. D. Gonzalez-Diaz et al., Nucl. Instrum. Methods. A 555, 72 (2005)

51. R. Brun et al., Simulation program for particle physics experiments, GEANT: user guide and reference manual (CERN, Geneva, 1978)

52. A. Apostolou et al., J. Phys. Conf. Ser. 1024, 012013 (2018)

53. A. Zinchenko, et al., A vector-finding approach to track reconstruction in cbm much. CBM Progress Report 2014, edited by V. Friese, and C. Sturm, (GSI, Darmstadt, 2015), 116

54. S. Jowzaee et al., Eur. Phys. J. A 52, 7 (2016)

55. M. Abdel-Bary et al., Eur. Phys. J. A 46, 27 (2010)

56. J. Adamczewski-Musch et al., Phys. Rev. C 95, 015207 (2017)

57. G. Hohler, Landolt-Bornstein - Group I Elementary Particles, Nuclei and Atoms. pp. 7-8 (1983)

58. J. Adamczewski-Musch et al., Phys. Lett. B 781, 735 (2018)

59. I. Zychor et al., Phys. Lett. B 660, 167 (2008)

60. G. Agakishiev et al., Phys. Rev. C 87, 025201 (2013)

61. V. Flaminio, et al., (1984), eprint: CERN-HERA-84-01

62. I. Fröhlich, et al., PoS ACAT2007, 076 (2007)

63. A. Paszke, et al., NIPS Autodiff Workshop (2017)

64. W. Czyzycki, et al., Electromagnetic Calorimeter for HADES, 2011. arXiv:1109.5550 [nucl-ex] 